\title{
Effect of hemolysis on Fourier transform infrared and Raman spectra of blood plasma
}

\author{
Dinesh Medipally \\ Technological University Dublin, dinesh.medipally@tudublin.ie \\ Daniel Cullen \\ Technological University Dublin, Daniel.Cullen@TUDublin.ie \\ Valérie Untereiner \\ Universite de Reims Champagne-Ardenne
}

See next page for additional authors

Follow this and additional works at: https://arrow.tudublin.ie/radart

Part of the Oncology Commons, and the Radiation Medicine Commons

\section{Recommended Citation \\ Medipally DKR, Cullen D, Untereiner V, Bryant J, Sockalingum GD, Nguyen TNQ, Noone E, Bradshaw S, Finn $M$, Dunne M, Shannon AM, Armstrong J, Meade AD, Lyng FM. Effect of hemolysis on Fourier transform infrared and Raman spectra of blood plasma. J Biophotonics. 2020 Jul;13(7):e201960173. doi: 10.1002/ jbio.201960173. Epub 2020 Apr 7. PMID: 32162465.}

This Article is brought to you for free and open access by the Radiation and Environmental Science Centre at ARROW@TU Dublin. It has been accepted for inclusion in Articles by an authorized administrator of ARROW@TU Dublin. For more information, please contact arrow.admin@tudublin.ie, aisling.coyne@tudublin.ie, gerard.connolly@tudublin.ie.

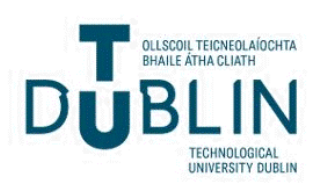




\section{Authors}

Dinesh Medipally, Daniel Cullen, Valérie Untereiner, Jane Bryant, Ganesh D. Sockalingum, Thi Nguyet Que Nguyen, Emma Noone, Shirley Bradshaw, Marie Finn, Mary Dunne, Aoife M. Shannon, John Armstrong, Aidan Meade, and Fiona Lyng 


\title{
Effect of haemolysis on FTIR and Raman spectra of blood plasma
}

Dinesh K.R. Medipally ${ }^{1,2}$, Daniel Cullen ${ }^{1,2}$, Valérie Untereiner ${ }^{3,4}$, Jane Bryant ${ }^{1}$, Ganesh D Sockalingum

${ }^{3}$, Thi Nguyet Que Nguyen ${ }^{1,2}$, Emma Noone ${ }^{5}$, Shirley Bradshaw ${ }^{5}$, Marie Finn ${ }^{5}$, Mary Dunne ${ }^{5}$, Aoife M. Shannon ${ }^{6}$, John Armstrong ${ }^{6,7}$, Aidan D Meade ${ }^{1,2, * *}$ and Fiona M. Lyng ${ }^{1,2, \uparrow *}$

1 Radiation and Environmental Science Centre, Focas Research Institute, Technological University Dublin, Dublin, D08 NF82, Ireland

2 School of Physics \& Clinical \& Optometric Sciences, Technological University Dublin, Dublin, D08 NF82, Ireland

3 BioSpecT EA 7506, Université de Reims Champagne-Ardenne, UFR Pharmacie, 51097 Reims, France

4 Plateforme en Imagerie Cellulaire et Tissulaire (PICT), Université de Reims Champagne-Ardenne, 51097 Reims, France

5 Clinical Trials Unit, St Luke's Radiation Oncology Network, St Luke's Hospital, Dublin, Dublin 6, Ireland

6 Cancer Trials Ireland, Dublin, D11 KXN4, Ireland

7 Department of Radiation Oncology, St Luke's Radiation Oncology Network, St Luke's Hospital, Dublin, Dublin 6, Ireland

* Correspondence: Fiona.lyng@dit.ie and Aidan.Meade@dit.ie

$\dagger$ Both authors contributed equally

\begin{abstract}
Haemolysis is a very common phenomenon and is referred as the release of intracellular components from red blood cells to the extracellular fluid. Haemolysed samples are often rejected in clinics due to the interference of haemoglobin and intracellular components in laboratory measurements. Plasma and serum based vibrational spectroscopy studies are extensively applied to generate spectral biomarkers for various diseases. However, no studies have reported the effect of haemolysis in blood based vibrational spectroscopy studies. This study was undertaken to evaluate the effect of haemolysis on infrared and Raman spectra of blood plasma. In this study, prostate cancer plasma samples $(n=30)$ were divided into three groups (non-haemolysed, mildly haemolysed and moderately hemolysed) based on the degree of haemolysis and FTIR and Raman spectra were recorded using high throughput (HT)-FTIR and HT-Raman spectroscopy. Discrimination was observed between the infrared and Raman spectra of non-haemolysed and haemolysed plasma samples using principal component analysis. A classical least square fitting analysis showed differences in the weighing of pure components in non-haemolysed and haemolysed plasma samples. Therefore, it is worth to consider the changes in spectral features due to haemolysis when comparing the results within and between experiments.
\end{abstract}


Keywords: Haemolysis, blood plasma, FTIR spectroscopy, Raman spectroscopy, principal component analysis, classical least squares fitting analysis.

\section{Introduction}

Haemolysis (or hemolysis) refers to the breakdown of erythrocytes and resulting in the release of intracellular components into the extracellular fluid, i.e. the plasma or serum [1] . Haemolysis is discernible as a red colouration of the plasma or serum after centrifugation of the sample and can occur both in-vivo and in vitro. The upper reference limit for free haemoglobin in plasma is around $20 \mathrm{mg} / \mathrm{L}$ [2]. Several factors causing in-vitro haemolysis are given in Table 1 [2]. Not all haemolysed specimens can be associated with in-vitro haemolysis because more than 50 medical conditions are associated with in-vivo haemolysis [3], such as cancer [4], autoimmune disorders [5], surgery [6] etc. In-vivo haemolysis can be easily concealed by in-vitro haemolysis. In these cases, samples haemolysed because of a medical condition may be refused because haemolysis had been attributed to improper specimen phlebotomy, without considering the condition causing in-vivo haemolysis [7].

In-vivo haemolysis can be distinguished from in-vitro haemolysis by estimating the concentration of haptoglobin [8]. Haptoglobin is formed in the liver and binds to free haemoglobin released from in-vivo lysed red cells to prevent its toxic effects [9]. The haptoglobin levels are reduced in the presence of large amounts of free haemoglobin, a decrease in the concentration of serum haptoglobin is the most marked laboratory indicator of in-vivo haemolysis [9]. In severe cases of in-vivo haemolysis, haptoglobin levels may be very low or even undetectable, whereas with invitro haemolysis the concentration of haptoglobin is usually not affected [8]. However, several studies have examined the potential of haptoglobin as a marker of in-vivo haemolysis. Varying degrees of sensitivity and specificity for haptoglobin have been shown depending on the cutoff concentration used. One study used a haptoglobin cutoff of less than $25 \mathrm{mg} / \mathrm{dL}$ and demonstrated $83 \%$ sensitivity and $96 \%$ specificity for intravascular haemolysis [10]. Another used a haptoglobin cutoff of less than $28 \mathrm{mg} / \mathrm{dL}$ and noted $91.8 \%$ sensitivity and $98.4 \%$ specificity for intravascular haemolysis [11]. On the other hand, decreased levels of haptoglobin can occur in the absence of haemolysis, due to the liver cirrhosis, disseminated ovarian carcinomatosis, pulmonary sarcoidosis, and elevated oestrogen states [9]. 
In general, there is no gold standard test to confirm in-vivo haemolysis, and most often labs rely on other clinical factors (e.g. increased reticulocyte count and decreased haptoglobin) and correlation with other laboratory markers (e.g., complete blood count, lactate dehydrogenase and indirect bilirubin) in addition to a patient's history [2,9].

Many laboratory chemistry tests depend on the evaluation of light that passes through a sample, and therefore haemolysed blood can interfere with these tests, as the red colour of haemoglobin can interfere with the absorption of light as it passes through a haemolysed sample [12]. In spectrophotometric assays, the absorption peak of oxyhaemoglobin lies between 531 and $543 \mathrm{~nm}$. This absorption peak is relatively broad so haemolysis intervenes over many wavelengths that are used for the quantification of analytes [12]. Haemolysis also interferes with laboratory tests by releasing analytes into the serum or plasma that are elevated in concentration within the red blood cells compared to serum or plasma, thereby giving false concentrations of measured analytes [13].

The application of vibrational spectroscopy (infrared and Raman spectroscopy) to generate spectral biomarkers of disease using biofluids such as plasma or serum is gaining increased focus. Infrared spectroscopy is based on the absorption of infrared radiation by the sample under study and the fact that molecules absorb specific frequencies of the incident light which are characteristic of their structure. Raman spectroscopy is based on inelastic scattering of monochromatic light, usually from a laser source. Many articles have been published reporting the use of Fourier Transform Infrared (FTIR) and Raman spectroscopy with serum and/or plasma as non-invasive diagnostics for various diseases, including cancers [14-17]. Despite haemolysis being very common in practice, no spectroscopic study has examined its effects on spectral markers and the concentration of analytes.

This study was undertaken to investigate the effect of haemolysis on Raman and FTIR spectra of plasma in prostate cancer patient plasma samples $(\mathrm{n}=30)$. A combination of ultraviolet (UV)Visible, high throughput (HT) - Raman and HT-FTIR spectroscopies were used to evaluate the spectral content of both haemolysed and non-haemolysed plasma. Significant spectral differences were observed in the infrared and Raman spectra of non-haemolysed and haemolysed plasma samples. The acquired FTIR and Raman spectra were also analysed by principal component analysis (PCA) and classical least squares (CLS) fitting analysis in the MATLAB environment. 
The CLS fitting analysis was employed to estimate the changes in the weighting of various analytes in non-haemolysed and haemolysed plasma samples.

\section{Experimental section}

\subsection{Ethical approval}

Ethical approval was awarded by the Technological University Dublin Research Ethics Committee for the collection of blood samples from healthy donors. The prostate cancer patients used in this study were recruited from the Cancer Trials Ireland (formerly All Ireland Cooperative Oncology Research Group, ICORG) trial 08-17 which is entitled "A Prospective Phase II Dose Escalation Study Using intensity modulated radiotherapy (IMRT) for High Risk N0 (cancer has not spread to nearby lymph nodes) M0 (or elsewhere in the body) Prostate Cancer (NCT00951535)". The patients were recruited for a translational study on vibrational spectroscopy for monitoring radiation therapy response [18]. The translational research study was approved by the St Luke's Radiation Oncology Network Research Ethics Committee and all research was performed in accordance with relevant guidelines and regulations. Informed consent was obtained from all participants. Fresh whole blood was drawn into Li-heparin tubes at St. Luke`s Radiation Oncology Network, St. Luke's Hospital (SLRON SLH), in Dublin, and were coded before being transferred to the Technological University (TU) Dublin laboratory. Plasma from prostate cancer patients were used to study the effect of in-vivo haemolysis on Raman and FTIR spectra of blood plasma. The clinical characteristics of prostate cancer patients are detailed in Table 2. Plasma from male healthy donor (age, 50 years) was used to study the effect of in-vitro haemolysis on Raman spectra of blood plasma.

\subsection{Plasma isolation}

Plasma was isolated from these blood samples by centrifugation at $3500 \mathrm{~g}$ for 5 minutes at $18^{\circ} \mathrm{C}$. The samples were subsequently stored at $-80^{\circ} \mathrm{C}$ prior to FTIR and Raman acquisition.

\subsection{Haemoglobin isolation}

A volume of $1 \mathrm{ml}$ of whole blood sample was centrifuged at $3500 \mathrm{~g}$ for 5 minutes to remove plasma. The pelleted red blood cells (RBCs) was purified by washing with phosphate buffer saline (PBS) 
for at least three times. The residual PBS was removed by centrifugation at $3500 \mathrm{~g}$ for 1 minute After purification, deionised water was added to the RBCs and sonication was performed for 30 sec to rupture the RBCs. Then, the diluted haemoglobin was further centrifuged at $3500 \mathrm{~g}$ for 5 minutes to remove $\mathrm{RBC}$ membrane. The isolated diluted hemoglobin sample was stored at $-80^{\circ} \mathrm{C}$ before Raman measurements.

\subsection{Ultraviolet - visible spectroscopy}

UV-visible spectroscopy is generally used in analytical chemistry for the quantitative determination of various analytes. UV-visible spectroscopy uses light in the visible and adjacent ranges. In this study, free haemoglobin of the plasma samples was measured spectrophotometrically (Perkin Elmer Lambda 900) with water as a blank. Thirty microliters of plasma samples were deposited on the quartz microsample cell with $0.1 \mathrm{~mm}$ pathlength and the absorbance at $414 \mathrm{~nm}$ were used to determine the haemolysis in the sample. The concentration of haemoglobin in plasma was determined using the Beer-Lambert law.

\subsection{FTIR spectroscopy}

The sample preparation and acquisition methodology for FTIR spectra was detailed in our previous paper [18]. Briefly, plasma samples stored at $-80^{\circ} \mathrm{C}$ were thawed at room temperature and were diluted threefold in physiological water. A volume of $4 \mu \mathrm{L}$ diluted plasma sample was deposited on a 384-well silicon plate (Bruker Optics GmbH, Ettlingen, Germany), and air-dried at room temperature. For each sample, 10 spots were used giving 10 instrumental replicates. The plate was then inserted into a high-throughput module (HTS-XT, Bruker Optics GmbH) attached to an FTIR spectrometer (Tensor 27, Bruker Optics GmbH). FTIR spectra were acquired in the transmission mode using the OPUS v6.5 software (Bruker Optics GmbH) in the wavenumber range from 4000 to $400 \mathrm{~cm}^{-1}$, using a spectral resolution of $4 \mathrm{~cm}^{-1}$ and 32 co-additions. FTIR spectra were then subjected to a quality test (OPUS v6.5) and details of this test are fully described in reference [19, 20]. Spectra that passed the quality test were pre-processed and processed in the wavenumber range from 800 to $4000 \mathrm{~cm}^{-1}$. 


\subsection{Raman spectroscopy}

Raman spectra from liquid plasma samples were recorded using an in house developed HT-Raman spectroscopy method [17]. Briefly, $20 \mu 1$ of liquid plasma samples were deposited on a cover glass bottomed 96 well plate (MatTek corporation) and Raman spectra of the plasma samples were acquired using a $785 \mathrm{~nm}$ laser focused through a 10x objective (N.A. 0.25). Spectra were recorded using a diffraction grating ruled with 300 lines $/ \mathrm{mm}$ giving a spectral resolution of $\sim 2.1 \mathrm{~cm}^{-1}$. Spectra were recorded automatically from each well where the spectrometer was programmed using an in-house developed high throughput macro template. Each spectrum was acquired over the region from $400-1800 \mathrm{~cm}^{-1}$. Ten spectra were recorded from each sample for each patient with a $20 \mathrm{~s}$ x 2 integration time. Multiple wavenumber calibration spectra of 1,4-Bis (2-methylstyryl) benzene and intensity calibration spectra of National Institute of Standards and Technology (NIST) Standard Reference Material (SRM) no. 2241 were recorded along with each sample acquisition and used in spectral post-processing.

\subsection{Pure molecular reference species recording}

All pure molecular references species (Table 3) were purchased from Sigma Aldrich. These molecular species include proteins, lipids and other analytes which are usually found in the blood plasma. Moreover, previous other studies also reported the variations in most of these molecular species due to the effect of haemolysis [13, 21-23]. Additionally, molecular reference species of lipids and fatty acids were included to study their interference in the plasma spectra due to in-vivo haemolysis. More details of these pure molecular reference species are provided in supplementary material.

Approximately 1-2 mg of each lyophilised analyte or $10 \mu \mathrm{L}$ of liquid analyte was deposited on a calcium fluoride slide and Raman spectra were recorded with a $785 \mathrm{~nm}$ laser excitation. The laser was focused through a $10 \times$ objective (N.A. 0.25 ) using a diffraction grating ruled with a grating of 300 lines per mm. Five spectra per sample were recorded for the acquisition time of 10 seconds and 2 accumulations. FTIR spectra of all analytes were recorded using Attenuated total reflectance (ATR)-FTIR (Perkin Elmer). Approximately $1 \mathrm{mg}$ of lyophilised analyte or $5 \mu \mathrm{L}$ of liquid analyte (allowed to dry at room temperature) was placed on the ATR crystal (Diamond / Zinc Selenide with refractive index 2.4) and FTIR spectra were acquired in the transmission mode using the 
spectrum software and using the following conditions: wavenumber range from 4000 to $700 \mathrm{~cm}^{-1}$, spectral resolution of $4 \mathrm{~cm}^{-1}$, and each spectrum was averaged over 8 scans.

\subsection{Data analysis}

All spectral processing procedures were carried out using MATLAB (R2017a; Mathworks Inc., Natick, MA), along with in-house developed algorithms and procedures available within the PLS Toolbox (v 8.0.2, Eigenvector Research Inc., Wenatchee, MA).

\subsubsection{Pre-processing}

Pre-processing of FTIR spectra includes baseline correction, calculation of second derivative spectra and vector normalisation. Baseline correction was performed using the rubberband

baseline subtraction [24]. Second derivative spectra were calculated using Savitsky-Golay algorithm [25] and a window length of 9 points. All spectra were standardized using vector normalization before analysis.

The acquired Raman spectra were wavenumber calibrated relative to an in-house standard of 1,4Bis (2-methylstyryl) benzene using in-house developed calibration procedures [26] and the instrument response correction was performed using the spectrum of NIST Standard Reference Material (SRM) no. 2241, according to the method described [27]. Briefly, SRM 2241 emits a broadband luminescence spectrum when illuminated with $785 \mathrm{~nm}$ Raman excitation laser. The shape of this luminescence spectrum is represented by a polynomial expression that relates the relative spectral intensity to the Raman shift. This polynomial along with a standard luminescence spectrum can be employed to determine the spectral intensity-response correction, which is unique to each Raman system. The derived instrument intensity response correction can be used to obtain Raman spectra that are corrected for instrument dependent artifacts [27]. The wavenumber and instrument response corrected Raman spectra were smoothed using a Savitzky-Golay filter. The background and baseline correction were performed using Extended Multiplicative Scattering Correction (EMSC). Then, spectra were standardized using vector normalization. An example of intensity corrected, and pre-processed liquid plasma spectrum is shown in Figure 1.

\subsubsection{Multivariate analysis}

Pre-processed FTIR and Raman spectra were further processed with PCA and CLS fitting analysis. 


\subsubsection{PCA}

PCA was performed with the similar approach as described earlier [18]. In brief, PCA is a commonly used method for multivariate data compression and visualization. It describes data variance by identifying a new set of orthogonal features, called principal components (PCs).

\subsubsection{CLS fitting analysis}

In this study, classical least squares (CLS) fitting analysis was performed on the second derivative FTIR spectra and vector normalised Raman spectra to estimate the relative fraction (a proxy for concentration) of reference spectra (of pure components) within a sample spectrum. The use of CLS fitting analysis to determine the relative concentrations of cellular components have been reported previously $[28,29]$. The pure components used in this study are given in Table 2. CLS is an exploratory method that assumes that any complex spectrum is the linear sum of contributions from spectra of pure components that contribute to the spectrum as described in the following equation [30]:

$$
S=a_{1} C_{1}+a_{2} C_{2}+\cdots+E
$$

Where $S$ represents a sample spectrum, $a 1$ and $a 2$ are component spectra and $C 1$ and $C 2$ are the weights or concentrations assigned to each component spectrum. In the case of a Raman or FTIR spectrum, not all contributing pure components are known. Therefore, an error or residual, $E$, in the fitting estimation will be observed that will equate to the difference between the sample spectrum and the sum of the component spectra:

$$
E_{j}=S_{j}-\left(a_{1 j} C_{1}+a_{2 j} C_{2}+a_{3 j} C_{3}\right)
$$

Where $E_{j}$ is the error associated with the intensity of the $\mathrm{j}$-th wavenumber. Applying CLS minimizes the error in fitting the sample spectrum, $S$. The minimum error occurs when the differential of the sum of squared errors is equal to zero.

$$
\frac{\left(d\left(\sum j E_{j}^{2}\right)\right.}{d C_{1}}=\frac{d \sum_{j}\left(S_{j}-\left(a_{1 j} C_{1}+a_{2 j} C_{2}+a_{3 j} C_{3}\right)^{2}\right)}{d C_{1}}=0
$$

Differentiating with respect to each of the component will give the above equation and using the method of simultaneous equations the weighting components can be calculated. Alternatively 
using matrix methods and the matrix of weighting coefficients can be solved for using the following equation.

$$
[C]=[A][a]^{T}\left[a a^{T}\right]^{-1}
$$

where $a$ is a component of sample spectra.

\section{Results and discussion}

\subsection{Haemoglobin spectra}

Three different types of plasma samples were observed in our plasma biobank (Figure 2). The normal plasma sample is clear and pale yellow in colour (Figure 2C). The haemolysed plasma sample is pale red in colour (Figure 2A) and the lipemic plasma sample is white in colour (Figure 2B). Lipemia is a turbidity of the sample caused by aggregation of lipoprotein particles ${ }^{31}$.

Figure 3 shows a representative UV-Visible absorption spectrum of haemoglobin recorded from the haemolysed prostate cancer plasma sample. The absorption bands at $414 \mathrm{~cm}^{-1}, 546 \mathrm{~cm}^{-1}$ and $571 \mathrm{~cm}^{-1}$ indicates the presence of oxyhaemoglobin. Figure 4 shows the FTIR absorption spectrum recorded from the haemoglobin standard (Sigma Aldrich). As haemoglobin is a protein, the FTIR absorption spectrum contains amide I band (between 1600 and $1700 \mathrm{~cm}^{-1}$ ), amide II band (between 1510 and $1580 \mathrm{~cm}^{-1}$ ), amide III band (between 1300 and $1400 \mathrm{~cm}^{-1}$ ), amide A band (between 3300 and $3500 \mathrm{~cm}^{-1}$ ). Figure 5A, 5B and 5C shows the Raman spectra recorded from the lyophilised haemoglobin standard, diluted haemoglobin standard and the haemoglobin isolated from blood respectively. The Raman spectra acquired from the lyophilised haemoglobin standard gave a fluorescence signal because of the bright red colour of haemoglobin. However, the fluorescence signal was not observed in Raman spectra from diluted and isolated haemoglobin. This might be due to the reduction of red colour of haemoglobin by diluting with deionised water. The Raman spectrum from diluted haemoglobin and isolated haemoglobin contains protein related bands at $750 \mathrm{~cm}^{-1}, 1002 \mathrm{~cm}^{-1}, 1035 \mathrm{~cm}^{-1}, 1125-1150 \mathrm{~cm}^{-1}, 1210 \mathrm{~cm}^{-1}$ (phenylalanine), $1170 \mathrm{~cm}^{-1}$ (tyrosine), $1035 \mathrm{~cm}^{-1}, 1230 \mathrm{~cm}^{-1}-1300 \mathrm{~cm}^{-1}$ (Amide III), $1480 \mathrm{~cm}^{-1}-1580 \mathrm{~cm}^{-1}$ (Amide III), and 1600 -

$1660 \mathrm{~cm}^{-1}$ (phenylalanine, tyrosine, Amide I). These bands are more prominent in diluted haemoglobin compared to isolated haemoglobin from RBC. 


\subsection{Plasma categorization based on haemolysis}

Specimens were categorized according to the haemoglobin concentration into three groups:

- Group $1(0$ - 0.1g/L), non-haemolysed ( $\mathrm{n}=12)$;

- Group $2(0.20-0.50 \mathrm{~g} / \mathrm{L})$, mildly haemolysed $(\mathrm{n}=10)$;

- Group $3(0.50-0.93 \mathrm{~g} / \mathrm{L})$, moderately haemolysed $(\mathrm{n}=8)$.

The results were analysed to determine if non-haemolysed, mildly haemolysed and moderately haemolysed samples had a significant impact on the analyte concentrations estimated by CLS fitting analysis. The median free haemoglobin concentrations for groups 1,2 and 3 were measured as $0.02,0.44$ and $0.71 \mathrm{~g} / \mathrm{L}$ respectively. At free haemoglobin concentration of $0.2 \mathrm{~g} / \mathrm{L}$, haemolysis was visible by the light red colour of the plasma. The haemolysed plasma samples appeared to be more turbid compared to non-haemolysed plasma samples. The increase in turbidity in haemolysed samples may be due to hyperlipidemia. It has been reported that hyperlipidemia is associated with increased haemolysis [32]. It is postulated that enhanced lipid concentration changes the lipid content of the erythrocyte membrane, resulting in increased erythrocyte fragility and subsequent leakage of cellular content into the plasma [32].

\subsection{Comparison of spectral features of non-haemolysed and haemolysed plasma samples}

Figure 6 shows the mean FTIR spectra of non-haemolysed and haemolysed plasma obtained from the prostate cancer patients. To explain the differences between each group, difference spectra were computed by subtracting the spectra of haemolysed plasma samples from the spectra of nonhaemolysed plasma samples. The first panel in Figure 7 shows the difference FTIR spectra obtained from moderately haemolysed and mildly haemolysed plasma samples. The second panel shows the difference FTIR spectra obtained from mildly haemolysed and non-haemolysed plasma samples and the third panel shows the difference FTIR spectra obtained from moderately haemolysed and non-haemolysed plasma samples.

Major differences were observed in the region around $1550 \mathrm{~cm}^{-1}$ (Amide II) [20], $1660 \mathrm{~cm}^{-1}$ (Amide I) [20], 1740-1760 $\mathrm{cm}^{-1}$ (stretching vibrations of $(\mathrm{C}=\mathrm{O})$ of fatty acids, triglycerides, and 
cholesterol esters) [33], 2800-2950 $\mathrm{cm}^{-1}$ (stretching vibrations of $\left(\mathrm{CH}_{2} / \mathrm{CH}_{3}\right)$ of lipids fatty acids, triglycerides and proteins) [33], $3300 \mathrm{~cm}^{-1}$ (Amide A) [20] and 3400-3600 $\mathrm{cm}^{-1}$ (OH stretch) [34] between non-haemolysed and haemolysed plasma groups. The increase in fatty acids (1740-1760 $\mathrm{cm}^{-1}$ ), lipids $\left(2800-2960 \mathrm{~cm}^{-1}\right)$ and $\mathrm{OH}$ stretch $\left(3400-3600 \mathrm{~cm}^{-1}\right)$ and the decrease in glycogen, nucleic acids $\left(1000-1100 \mathrm{~cm}^{-1}\right)[33,35]$, amide II $\left(1550 \mathrm{~cm}^{-1}\right)$, amide I $\left(1660 \mathrm{~cm}^{-1}\right)$ and amide A $\left(3300 \mathrm{~cm}^{-1}\right)$ were observed with an increase in haemolysis. The decrease in amide II, amide I and amide A suggests the decrease in protein content with an increase in haemolysis and the increase in fatty acids, lipids and $\mathrm{OH}$ stretch suggest the increase in lipids with an increase in haemolysis. The increase in lipids and fatty acids in haemolysed plasma samples may indicate in-vivo haemolysis [32]. As mentioned earlier, the haemolysed plasma specimens appeared to be more turbid compared to non-haemolysed plasma samples due to hyperlipidemia. The increase in lipid concentration with an increase in haemolysis has also been reported previously [32].

Figure 8 shows the mean Raman spectra of non-haemolysed and haemolysed plasma obtained from the prostate cancer patients. The first panel in Figure 9 shows the difference Raman spectra obtained from moderately haemolysed and mildly haemolysed plasma samples. The second panel shows the difference Raman spectra obtained from mildly haemolysed and non-haemolysed plasma samples and the third panel shows the difference Raman spectra obtained from moderately haemolysed and non-haemolysed plasma samples. Major intensity related differences were observed in the region around $877 \mathrm{~cm}^{-1}, 881 \mathrm{~cm}^{-1}, 1006 \mathrm{~cm}^{-1}, 1085 \mathrm{~cm}^{-1}, 1210 \mathrm{~cm}^{-1}, 1270 \mathrm{~cm}^{-1}$, $1302 \mathrm{~cm}^{-1}, 1345 \mathrm{~cm}^{-1}, 1450 \mathrm{~cm}^{-1}, 1528 \mathrm{~cm}^{-1}$, and $1660 \mathrm{~cm}^{-1}$. The bands corresponding to phenylalanine $\left(1006 \mathrm{~cm}^{-1}\right.$ and $\left.1210 \mathrm{~cm}^{-1}\right)$ [36] increased with increase in haemolysis. The increase in the amide linkage bands $\left(1302,1340 \mathrm{~cm}^{-1}\right)$ [37] was observed in non-haemolysed plasma compared to haemolysed plasma. The $\mathrm{CH}_{2}$ deformation $\left(1340 \mathrm{~cm}^{-1}\right)$ [37] bands were decreased with increase in haemolysis. The bands corresponding to cholesterol $\left(881 \mathrm{~cm}^{-1}\right.$ and $\left.1450 \mathrm{~cm}^{-1}\right)$ [38] and triglycerides $\left(877 \mathrm{~cm}^{-1}, 1270 \mathrm{~cm}^{-1}\right.$ and $\left.1450 \mathrm{~cm}^{-1}\right)$ [38] were increased with increase in haemolysis. This increase in lipid profile with an increase in haemolysis was also observed in the FTIR spectral analysis. 


\subsection{Multivariate analysis}

\subsubsection{PCA}

PCA was performed on second derivative of the mean FTIR spectra by patient, using the wavenumber range of 4000-800 $\mathrm{cm}^{-1}$. PCA was carried out with 10 PCs and the first 2 PCs accounted for $\sim 91 \%$ of the total percentage variance. The first two PCs were used to visualize the classification between the groups. The scatter plot revealed three well differentiated clusters between the analysed groups (Figure 10A).

The PCA analysis showed clear discrimination between the FTIR spectra of non, mildly and moderately haemolysed plasma samples. PC1 showed the discrimination between the FTIR spectra of non and mild haemolysed plasma samples and moderate haemolysed plasma samples. PC1 shows positive band associated with proteins $\left(1657 \mathrm{~cm}^{-1}\right)$ in the moderately haemolysed plasma group and negative bands associated with glycogen, nucleic acids $\left(1040 \mathrm{~cm}^{-1}, 1063 \mathrm{~cm}^{-1}, 1090 \mathrm{~cm}^{-}\right.$

${ }^{1}$ and $\left.1120 \mathrm{~cm}^{-1}\right)$, lipids, triglycerides and fatty acids $\left(1744 \mathrm{~cm}^{-1}, 2853 \mathrm{~cm}^{-1}, 2926 \mathrm{~cm}^{-1}\right.$ and 2963 $\mathrm{cm}^{-1}$ ) in the non and mildly haemolysed plasma groups. PC2 showed the discrimination between the FTIR spectra of non-haemolysed and mild haemolysed plasma samples. PC2 shows positive bands at $1040 \mathrm{~cm}^{-1}, 1120 \mathrm{~cm}^{-1}$ and $1640 \mathrm{~cm}^{-1}$ in the mildly haemolysed plasma group and negative bands at $1744 \mathrm{~cm}^{-1}, 2853 \mathrm{~cm}^{-1}, 2926 \mathrm{~cm}^{-1}$ and $2963 \mathrm{~cm}^{-1}$ in the non-haemolysed plasma group. The positive bands in PC2 are assigned to glycogen, RNA and protein and negative bands are assigned to lipids, triglycerides and fatty acids. The PCA analysis showed the main discriminating features between non-haemolysed and haemolysed plasma samples are related to glycogen, nucleic acids, proteins, fatty acids, triglycerides and lipids. The FTIR difference spectrum analysis also showed similar discriminating spectral features between the non-haemolysed, mild and moderate haemolysed plasma samples.

Figure 11 shows the results of PCA analysis performed on the vector normalised Raman spectra of non-haemolysed and haemolysed plasma groups. The first two PCs accounted for $\sim 58 \%$ of the total percentage variance and the scatter plot revealed overlapped clusters between the analysed groups. Only PC1 showed the discrimination between the Raman spectra of non and mild haemolysed plasma samples and moderate haemolysed plasma samples. PC1 has positive bands at $1003 \mathrm{~cm}^{-1}, 1080 \mathrm{~cm}^{-1}, 1155 \mathrm{~cm}^{-1}, 1430 \mathrm{~cm}^{-1}$ and $1650 \mathrm{~cm}^{-1}$ in the non and mildly haemolysed 
plasma groups and negative bands at $1007 \mathrm{~cm}^{-1}, 1210 \mathrm{~cm}^{-1}, 1260 \mathrm{~cm}^{-1}, 1346 \mathrm{~cm}^{-1} 1460 \mathrm{~cm}^{-1}$ and $1660 \mathrm{~cm}^{-1}$ in the moderately haemolysed plasma group. The positive bands assigned to proteins, amino acids, carotenoids and lipids, and negative bands related to amino acids, proteins and lipids. The PCA showed proteins and lipids are the major discriminating features between the Raman spectra of plasma from non and mildly haemolysed versus moderately haemolysed patient groups. Similar discriminating features were also observed in the difference spectrum analysis of Raman spectra from non-haemolysed and haemolysed plasma groups.

\subsubsection{CLS fitting analysis}

CLS fitting analysis was performed to examine the alterations in the weighting of analytes in Raman and FTIR spectra with variable haemolysis. Significance testing was performed on the identified plasma analytes using two-tailed students t-tests with the significance level of $\mathrm{p} \leq 0.05$, 0.01 and 0.001 . Figure 12 shows the results of CLS fitting analysis performed on the nonhaemolysed and haemolysed plasma second derivative FTIR spectra. In particular a differentiation in the weighting of albumin, phosphatidyl-ethanolamine ( $\mathrm{p}$-ethanolamine), uric acid, ceramide, thymidine, ubiquitin, triglyceride, Interleukin-6 and Interleukin- 8 were observed between the FTIR spectra of non-haemolysed and haemolysed plasma samples. An increase in the weighting of albumin, ceramide, uric acid and ubiquitin was observed with an increase in haemolysis. Conversely a decreased weighting of thymidine and interleukin- 6 was observed with increase in haemolysis. Similarly, a decreased weighting of triglyceride was observed in the haemolysed samples compared to non-haemolysed plasma samples. The increase in ceramide and pethanolamine suggests an increase in lipid concentration with increase in haemolysis. An increased concentration of albumin [13] and ubiquitin [39] with an increase in haemolysis has also been reported previously.

Figure 13 shows the results of CLS fitting analysis performed on the Raman spectra from nonhaemolysed and haemolysed plasma. Significant differences were observed in the weighting of uric acid, albumin, insulin, p-ethanolamine, creatinine, interleukin-6, RNA, urea and thymidine. A decreased weighting for uric acid and urea was observed with increase in haemolysis. A decreased concentration of uric acid with increase in haemolysis has also been reported previously [21] and may arise from a premature decomposition of haemoglobin [21]. Conversely an increased 
weighting of RNA and Interleukin-6 was observed with increase in haemolysis. Kirschner et al. reported an increase in the micro RNA levels in haemolysed plasma specimens [23]. The weighting of insulin within Raman spectra was decreased in mildly haemolysed samples and increased in moderately haemolysed plasma samples. The weighting of albumin and creatinine was decreased in moderately haemolysed samples compared to non and mildly haemolysed plasma samples.

Variations in the weighting of albumin, uric acid, p-ethanolamine, interleukin- 6 and thymidine were observed in both FTIR and Raman spectra of non-haemolysed and haemolysed plasma samples. Increased weighting of p-ethanolamine was observed in both FTIR and Raman spectral analysis. The FTIR spectral analysis showed an increased weighting of albumin with an increase in haemolysis but a slightly decreased level of albumin was observed in the Raman spectra of the moderately haemolysed plasma samples. A decreased weighting of uric acid, thymidine and interleukin-6 was observed in the FTIR spectral analysis with an increase in haemolysis. Conversely, an increased weighting of uric acid, thymidine and interleukin-6 levels were observed in the Raman spectral analysis. The differences in the CLS fitting analysis results of FTIR and Raman spectra may be due to the employment of different pre-processing and sample preparation methods. Second derivative FTIR spectra were used in this analysis, second derivative spectra allow more distinct identification of small and adjacent lying absorption peaks which are not clearly visible in the original spectrum. The FTIR spectra were recorded from diluted and dried plasma samples and Raman spectra were recorded from liquid plasma samples. Differences in the sample preparation methods might also affect the identification of plasma analytes. However, further studies would be required to confirm the variation in plasma analytes due to the different sample preparation methods.

\section{Interpatient variability}

The above studies were carried out on plasma samples obtained from various prostate cancer patients. To avoid any interpatient variability, plasma from a healthy control subject was spiked with increasing concentrations of diluted haemoglobin (extracted from whole blood), $0.44 \mathrm{~g} / \mathrm{L}$ (mildly haemolysed), $0.93 \mathrm{~g} / \mathrm{L}$ (moderately-haemolysed) and $1.31 \mathrm{~g} / \mathrm{L}$ (grossly-haemolysed). Figure 14 shows the mean spectra of non-haemolysed and in-vitro haemolysed plasma samples. Intensity related differences were observed across the spectra in the analysed plasma groups. A decrease in the bands around $1007 \mathrm{~cm}^{-1}, 1450 \mathrm{~cm}^{-1}$ and $1660 \mathrm{~cm}^{-1}$ were observed with an increase 
in in-vitro haemolysis. These spectral features were increased with an increase in haemolysis in the Raman spectra of plasma from prostate cancer patients and could originate in in-vivo haemolysis or interpatient variability. Figure 15 shows the PCA scatter plot for non-haemolysed and in-vitro spiked-haemolysed plasma samples. The first two PC's accounted for $\sim 25 \%$ of the total percentage variance and the scatter plot revealed overlapped clusters between the analysed groups. This suggests that there are no significant discriminating spectral features between the non-haemolysed and in-vitro haemolysed plasma specimens.

The CLS fitting analysis performed on the in-vitro haemolysed plasma samples showed differences in the weighting of uric acid, albumin, insulin, p-ethanolamine, Interleukin-6, RNA, urea and thymidine (Figure 16). A decrease in insulin concentration was observed with an increase in haemolysis. This might be attributable to the previously observed release of proteolytic enzymes during hemolysis that destroys small peptides such as insulin, gastrin etc [22]. However, this trend was not observed in prostate cancer plasma samples. The increased concentration of urea in haemolysed samples was reported previously [40] and this effect might be due to the cellular release of urea into plasma during haemolysis [40]. However, in this study, the concentration of urea was increased gradually from non-haemolysed through mildly haemolysed up to moderately haemolysed samples and then decreased in grossly haemolysed plasma samples. Conversely, a decreased weighting of urea was observed in prostate cancer plasma samples with an increase in haemolysis. The decreased levels of uric acid [13], creatinine [13], insulin [22] and increased levels of urea [40], micro RNA [23] and interleukins [41] were reported with an increase in haemolysis. Similar trends were observed in this study up to moderately haemolysed samples but a deviation in this trend was observed in grossly haemolysed samples. This might be due to dilution or to interference from the bright red colour of haemoglobin as more haemoglobin was added to the plasma to produce the grossly haemolysed plasma sample. This results in dilution and alteration of analytes present in the plasma sample. Previous studies have also reported variations in severely haemolysed plasma samples due to dilution $[13,40]$.

The weighting of uric acid, Interleukin- 6 and RNA were consistent in both in-vitro haemolysed and prostate cancer plasma samples and the weighting of urea, insulin, p-ethanolamine, thymidine and creatine were inconsistent between the in-vitro and prostate cancer plasma samples. Consistent weighting of pure components in in-vitro haemolysed samples and prostate cancer haemolysed 
plasma samples suggests the interference of haemoglobin colour and intercellular components of $\mathrm{RBC}$ in the weighing of plasma analytes. Inconsistent weighting of pure components in in-vitro haemolysed and prostate cancer haemolysed plasma samples might be due to in-vivo haemolysis or due to the development of cancer.

\section{Conclusions}

Haemolysis, in-vitro or in-vivo, can affect the concentration of analytes present in the blood plasma and serum. Haemolysis can interfere in the quantification of several analytes in three different ways. The first one is the interference of the red coloration of haemoglobin with the absorption of light as it passes through a sample. The second interference is by releasing analytes into the serum or plasma that are in high concentration within the red blood cells and the third interference is by increasing lipid and fatty acid concentration in in-vivo haemolysis. Many studies have reported the interference of haemoglobin in various biochemical assays, including UV-visible spectroscopy. This study aimed to investigate the changes in the IR and Raman spectra of nonhaemolysed and haemolysed plasma samples. The mean spectra showed more changes in the FTIR spectral features of non-haemolysed and haemolysed patient plasma groups compared to the Raman spectral features. The increased haemolysis with an increase in the lipid content suggests an in-vivo haemolysis in the prostate cancer plasma samples. The PCA analysis performed on FTIR spectra showed a good separation of the plasma groups compared to the PCA performed on Raman spectra. This suggest that FTIR spectroscopy is a preferred method for the analysis of haemolysed samples. Finally, the CLS fitting analysis suggested significant alterations in the weighing of analytes in FTIR and Raman spectra of non-haemolysed and haemolysed patient plasma groups. The Raman spectroscopy study on in-vitro haemolysed samples showed minor changes in the spectral features of non-haemolysed and haemolysed plasma specimens and CLS fitting analysis showed similar changes in the weighting of some pure components as observed in the Raman spectra of plasma from prostate cancer patients.

This study suggests that the FTIR spectra show more significant changes in the spectral features and in the weighting of pure components due to haemolysis compared to the Raman spectra. However, further FTIR studies should be performed on in-vitro haemolysed samples to confirm these results. Both in-vitro and in-vivo haemolysis is a common phenomenon and rejecting these 
samples could limit the diagnostic performance of Raman and FTIR spectroscopy. However, when including haemolysed samples in clinical spectroscopy studies, it is important to consider any changes in FTIR and Raman spectral features due to haemolysis when comparing results within and between experiments. Differences in the spectral features due to haemolysis will be observed within the patient group but most likely, these differences will be minor when compared between patient groups, for example control and cancer cases. However, further studies are needed to confirm this.

This is the first study to report the effect of haemolysis on infrared and Raman spectra of blood plasma. This study was performed on a limited sample set and validation of these results on a larger cohort will be necessary to confirm the effect of in-vitro and in-vivo haemolysis on FTIR and Raman spectra of blood plasma. Future work should also involve biological assays showing proteins, lipids, circulating nucleic acids and other analyte levels to corroborate the findings of the CLS fitting analysis.

Funding: This research was funded by an Irish Research Council Postgraduate Award, COST Action (BM1401) Short Term Scientific Mission Award, Irish Research Council-Ulysses Award (Grant Number PJ29007), EU FP7 Network of Excellence DoReMi (Grant Number 249689) and Science Foundation Ireland (11/RFP.1/BMT/3317).

Acknowledgements: Cancer Trials Ireland acted as sponsor for the CTRIAL-IE (ICORG) 08-17 study, providing project management, protocol development and monitoring, patient information leaflet and CRF development, ethics application and amendments. The authors are very grateful to the patients who participated in the CT-IE 08-17 study and donated blood samples for this translational research study.

Conflicts of interest: The authors declare no conflicts of interest. 


\section{References}

(1) Lippi, G., Blanckaert, N., Bonini, P., Green, S., Kitchen, S., Palicka, V., Vassault, A. J., Plebani, M. Haemolysis: An Overview of the Leading Cause of Unsuitable Specimens in Clinical Laboratories. Clinical Chemistry and Laboratory Medicine. 2008, 46(6), 764-72.

(2) Thomas, K. L. Haemolysis as Influence and Interference Factor. J. Int. Fed. Clin. Chem. Lab. Med. 2002, 13 (4), 95-98.

(3) Carraro, P., Servidio, G., Plebani, M. Hemolyzed Specimens: A Reason for Refection of a Clinical Challenge? Clin Chem. 2000, 46 (2), 306-307.

(4) Rytting, M., Worth, L., Jaffe, N. Hemolytic Disorders Associated with Cancer. Hematol Oncol Clin North Am. 1996, 10 (2), 365-376.

(5) Go, R. S., Winters, J. L., Kay, N. E. How I Treat Autoimmune Hemolytic Anemia. Blood. 2017, 129(22), 2971-2979.

(6) Vercaemst, L. Hemolysis in Cardiac Surgery Patients Undergoing Cardiopulmonary Bypass: A Review in Search of a Treatment Algorithm. J. Extra. Corpor. Technol. 2008, 40(4), 257-267.

(7) Howanitz, P. J., Lehman, C. M., Jones, B. A., Meier, F. A., Horowitz, G. L. Practices for Identifying and Rejecting Hemolyzed Specimens Are Highly Variable in Clinical Laboratories. Arch. Pathol. Lab. Med. 2015, 139(8), 1014-9.

(8) Lippi, G., Cervellin, G., Favaloro, E.J., Plebani, M. In Vitro and In Vivo Hemolysis - An Unresolved Dispute in Laboratory Medicine; Germany: Walter de Gruyter GmbH, 2012.

(9) Shih, AW., Mcfarlane, A., Verhovsek, M. Haptoglobin Testing in Hemolysis: Measurement and Interpretation. Am. J. Hematol. 2014. 89(4), 443-7.

(10) Marchand, A., Galen, R. S., Van Lente, F. The Predictive Value of Serum Haptoglobin in Hemolytic Disease. JAMA. 1980, 243(19), 1909-11.

(11) Kormoczi, G.F., Saemann, M.D., Buchta, C., Peck-Radosavljevic, M., Mayr, W.R., Schwartz, D.W.M., Dunkler, D., Spitzauer, S., Panzer, S. Influence of Clinical Factors on the Haemolysis Marker Haptoglobin. Eur. J. Clin. Invest. 2006, 36(3), 202-9. 
(12) Howanitz, P. J., Lehman, C. M., Jones, B. A., Meier, F. A., Horowitz, G. L. Clinical Laboratory Quality Practices When Hemolysis Occurs. Arch. Pathol. Lab. Med. 2015, 139(7), 901-6.

(13) Koseoglu, M., Hur, A., Atay, A., Cuhadar, S. Effects of Hemolysis Interference on Routine Biochemistry Parameters. Biochem. Medica. 2011, 21(1), 79-85.

(14) Sahu, A., Sawant, S., Mamgain, H., Krishna, C. M. Raman Spectroscopy of Serum: An Exploratory Study for Detection of Oral Cancers. Analyst 2013, 138, 4161-4174.

(15) Blat, A.; Wiercigroch, E.; Smeda, M.; Wislocka, A.; Chlopicki, S.; Malek, K. Fourier Transform Infrared Spectroscopic Signature of Blood Plasma in the Progression of Breast Cancer with Simultaneous Metastasis to Lungs. J. Biophotonics 2019, 12(10), e201900067.

(16) González-Solís, J. L.; Martínez-Espinosa, J. C.; Torres-González, L. A.; Aguilar-Lemarroy, A.; Jave-Suárez, L. F.; Palomares-Anda, P. Cervical Cancer Detection Based on Serum Sample Raman Spectroscopy. Lasers Med. Sci. 2014, 29 (3), 979-985.

(17) Medipally, D. K. R.; Maguire, A.; Bryant, J.; Armstrong, J.; Dunne, M.; Finn, M.; Lyng, F. M.; Meade, A. D. Development of a High Throughput (HT) Raman Spectroscopy Method for Rapid Screening of Liquid Blood Plasma from Prostate Cancer Patients. Analyst 2017, $142(8), 1216-1226$.

(18) Dinesh K.R. Medipally, Thi Nguyet Que Nguyen, Jane Bryant, Valérie Untereiner, Ganesh D. Sockalingum, Daniel Cullen, Emma Noone, Shirley Bradshaw, Marie Finn, Mary Dunne, Aoife M. Shannon, John Armstrong, F. M. L. and A. D. M. Monitoring Radiotherapeutic Response in Prostate Cancer Patients Using High Throughput FTIR Spectroscopy of Liquid Biopsies. Cancers (Basel). 2019, 11 (7), 925.

(19) Helm, D.; Labischinski, H.; Naumann, D. Elaboration of a Procedure for Identification of Bacteria Using Fourier-Transform IR Spectral Libraries: A Stepwise Correlation Approach. J. Microbiol. Methods. 1991, 14(2), 127-142.

(20) Lacombe, C.; Untereiner, V.; Gobinet, C.; Zater, M.; Sockalingum, G. D.; Garnotel, R. Rapid Screening of Classic Galactosemia Patients: A Proof-of-Concept Study Using HighThroughput FTIR Analysis of Plasma. Analyst 2015, 140 (7), 2280-2286. 
(21) Yucel, D.; Dalva, K. Effect of in Vitro Hemolysis on 25 Common Biochemical Tests. Clin. Chem. 1992, 38(4), 575-7.

(22) Jones, A. M.; Honour, J. W. Unusual Results from Immunoassays and the Role of the Clinical Endocrinologist. Clin. Endocrinol. (Oxf). 2006, 64(3):234-44.

(23) Kirschner, M. B.; Edelman, J. J. B.; Kao, S. C. H.; Vallely, M. P.; Van Zandwijk, N.; Reid, G. The Impact of Hemolysis on Cell-Free MicroRNA Biomarkers. Front. Genet. 2013, 24, 4,94 .

(24) Opus 5 Reference Manual; Bruker OPTIK GmbH, 2004.

(25) Savitzky, A.; Golay, M. J. E. Smoothing and Differentiation of Data by Simplified Least Squares Procedures. Anal. Chem. 1964, 36 (8), 1627-1639.

(26) Maguire, A.; Vegacarrascal, I.; White, L.; Mcclean, B.; Howe, O.; Lyng, F. M.; Meade, A. D. Analyses of Ionizing Radiation Effects In Vitro in Peripheral Blood Lymphocytes with Raman Spectroscopy. Radiat. Res. 2015, 183 (4), 407-416.

(27) Choquette SJ, Etz ES, Hurst WS, Blackburn DH, L. S. Relative Intensity Correction of Raman Spectrometers: NIST SRMs 2241 through 2243 for 785 Nm, 532 Nm, and 488 Nm/514.5 Nm Excitation. Appl. Spectrosc. 2007, 61 (2), 117-129.

(28) Notingher, I.; Jell, G.; Notingher, P. L.; Bisson, I.; Polak, J. M. P.; Hench, L. L. Raman Spectroscopy: Potential Tool for in Situ Characterization of Bone Cell Differentiation. Key Eng. Mater. 2005, 284-286, 545-548.

(29) Wu, Y.; Dong, Y.; Jiang, J.; Li, H.; Zhu, T.; Chen, S. Evaluation of the Bone-Ligament and Tendon Insertions Based on Raman Spectrum and Its PCA and CLS Analysis. Sci. Rep. $2017,7,38706$.

(30) Stanimirovic, O.; Boelens, H. F. M.; Mank, A. J. G.; Hoefsloot, H. C. J.; Smilde, A. K. Profiling of Liquid Crystal Displays with Raman Spectroscopy: Preprocessing of Spectra. Appl. Spectrosc. 2005, 59(3), 267-74.

(31) Nikolac, N. Lipemia: Causes, Interference Mechanisms, Detection and Management. Biochemia Medica. 2014, 4(1), 57-67. 
(32) Dimeski, G.; Mollee, P.; Carter, A. Increased Lipid Concentration Is Associated with Increased Hemolysis. Clinical Chemistry. 2005, 51(12), 2425.

(33) Staniszewska-Slezak, E.; Fedorowicz, A.; Kramkowski, K.; Leszczynska, A.; Chlopicki, S.; Baranska, M.; Malek, K. Plasma Biomarkers of Pulmonary Hypertension Identified by Fourier Transform Infrared Spectroscopy and Principal Component Analysis. Analyst. 2015, 140(7), 2273-9.

(34) Bonnier, F.; Baker, M. J.; Byrne, H. J. Vibrational Spectroscopic Analysis of Body Fluids: Avoiding Molecular Contamination Using Centrifugal Filtration. Anal. Methods. 2014, $6(14), 5155-5160$.

(35) Maziak, D. E.; Do, M. T.; Shamji, F. M.; Sundaresan, S. R.; Perkins, D. G.; Wong, P. T. Fourier-Transform Infrared Spectroscopic Study of Characteristic Molecular Structure in Cancer Cells of Esophagus: An Exploratory Study. Cancer Detect. Prev. 2007, 31(3), 24453.

(36) Gonzalez-Solís, J. L.; Martínez-Espinosa, J. C.; Salgado-Román, J. M.; Palomares-Anda, P. Monitoring of Chemotherapy Leukemia Treatment Using Raman Spectroscopy and Principal Component Analysis. Lasers Med. Sci. 2014, 29(3), 1241-9.

(37) Movasaghi, Z.; Rehman, S.; Rehman, I. U. Raman Spectroscopy of Biological Tissues. Applied Spectroscopy Reviews. 2007, 42(5), 493-541.

(38) Borges, R. de C. F.; Navarro, R. S.; Giana, H. E.; Tavares, F. G.; Fernandes, A. B.; Silveira, L. Detecting Alterations of Glucose and Lipid Components in Human Serum by NearInfrared Raman Spectroscopy. Rev. Bras. Eng. Biomed. 2015, 31(2), 160-168.

(39) Takada, K.; Nasu, H.; Hibi, N.; Tsukada, Y.; Shibasaki, T.; Fujise, K.; Fujimuro, M.; Sawada, H.; Yokosawa, H.; Ohkawa, K. Serum Concentrations of Free Ubiquitin and Multiubiquitin Chains. Clin. Chem. 1997, 43(7), 1188-95.

(40) Lippi, G.; Salvagno, G. L.; Montagnana, M.; Brocco, G.; Guidi, G. C. Influence of Hemolysis on Routine Clinical Chemistry Testing. Clin. Chem. Lab. Med. 2006, 44(3), $311-$ 6.

(41) Wei, J.; Zhao, J.; Schrott, V.; Zhang, Y.; Gladwin, M.; Bullock, G.; Zhao, Y. Red Blood 
Cells Store and Release Interleukin-33. J. Investig. Med. 2015. 63(6), 806-10. 


\section{Tables}

Table 1: Factors causing in vitro haemolysis [2].

\section{During specimen collection}

\section{After Specimen collection}

Strong aspiration while puncturing superficial Vigorous shaking of collected sample veins

Aspiration with large needles causes higher Centrifugation of sample before completion of haemolysis because of an increase in the flow- coagulation rate, flow speed and turbulence.

Partial blockage of a venous or arterial catheter Centrifugation of partially coagulated samples causes strong aspiration of the sample collected from patients on anticoagulants with a syringe.

\begin{tabular}{ll}
\hline $\begin{array}{l}\text { Specimen collection and subsequent splitting } \\
\text { into multiple tubes with a syringe. }\end{array}$ & $\begin{array}{l}\text { Positive or negative pressure in sample } \\
\text { collection tube }\end{array}$ \\
\hline & Dilution of blood with hypotonic solutions \\
\hline & Freeze thawing of whole blood \\
\hline & Storage or transportation of collected specimen \\
& over several days at ambient temperatures
\end{tabular}


Table 2: Clinical characteristics of prostate cancer patients used in the study.

\begin{tabular}{cccc}
\hline & Non-haemolysed & Mildly-haemolysed & Moderately-haemolysed \\
Number of subjects & 12 & 10 & 08 \\
\hline Sex & $\mathrm{M}$ & $\mathrm{M}$ & $\mathrm{M}$ \\
\hline Age (Years) & & & 74.1 \\
Mean & 70.4 & 67.1 & 74 \\
Median & 69.5 & 67 & $70-79$ \\
Range & $58-79$ & $58-76$ & 13.7 \\
PSA (ng/ml) & 14.38 & 17.9 & 9.8 \\
Mean & 10.95 & 7.57 & $6.8-26.5$ \\
Median & $1.55-40.04$ & $4-62$ & $01(12.5 \%)$ \\
Range & & $03(30 \%)$ & $07(87.5 \%)$ \\
T stage & $04(33.3 \%)$ & $07(70 \%)$ & $04(50 \%)$ \\
T2 & $08(66.6 \%)$ & & $02(25 \%)$ \\
T3 & $04(33.3 \%)$ & $04(40 \%)$ & $02(25 \%)$ \\
\hline 7
\end{tabular}


Table 3: Pure molecular reference species used in haemolysis study

\begin{tabular}{cc}
\hline Category & Pure components \\
\hline $\begin{array}{c}\text { Protein and related } \\
\text { compounds }\end{array}$ & $\begin{array}{c}\text { Apolipoprotein E4, Albumin, Haemoglobin } \\
\text { Interleukin-6, Interleukin-8 and Ubiquitin }\end{array}$ \\
\hline $\begin{array}{c}\text { Arachidonic acid, Cholesterol, Ceramide } \\
\text { Linolenic acid, Linoleic acid, }\end{array}$ & $\begin{array}{c}\text { (PUFA), } \\
\text { Sphingomyelin, } \\
\text { Lipids and Fatty acids }\end{array}$ \\
Oleic acid, Triglyceride, polyunsaturated fatty acid \\
phosphatidylcholine, phosphatidylethanolamine, \\
phosphatidylserine, prostaglandin E1, \\
phosphatidylinositol \\
related compounds
\end{tabular}




\section{Figures}

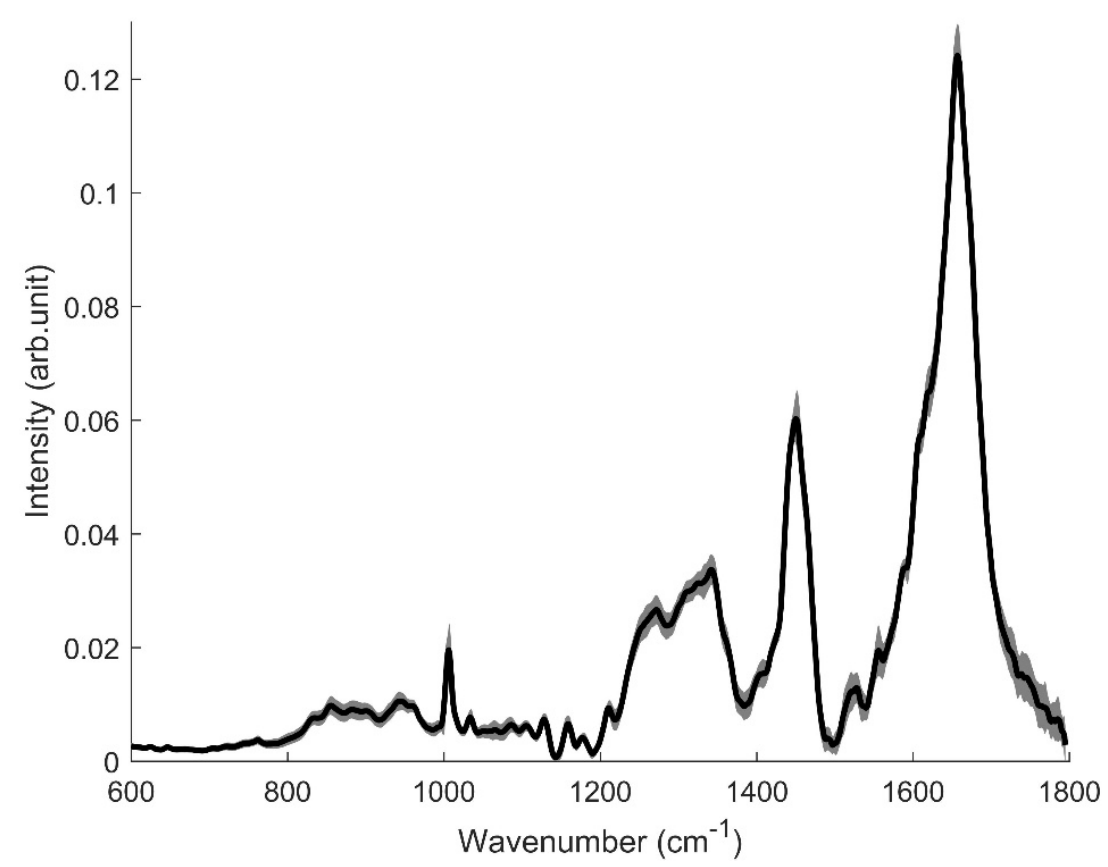

Figure 1: Mean and standard deviation of instrument response corrected and pre-processed liquid plasma Raman spectrum.

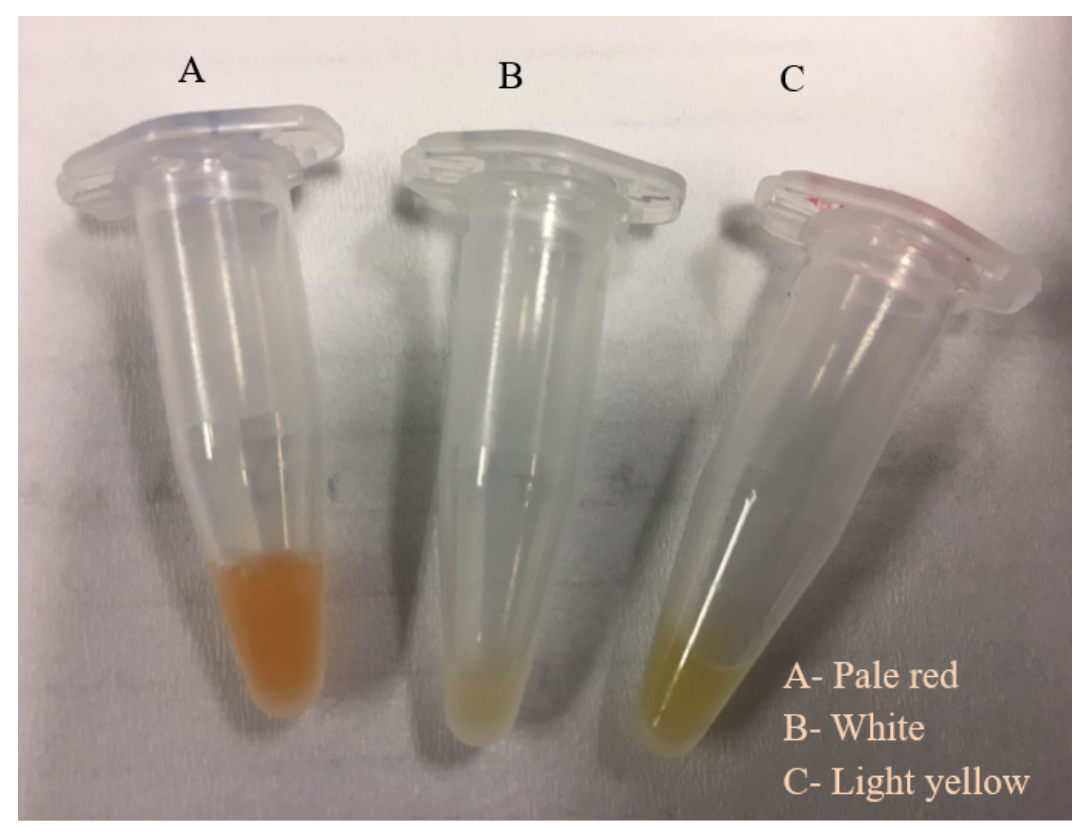

Figure 2: Three different types of liquid plasma samples in plasma biobank. 


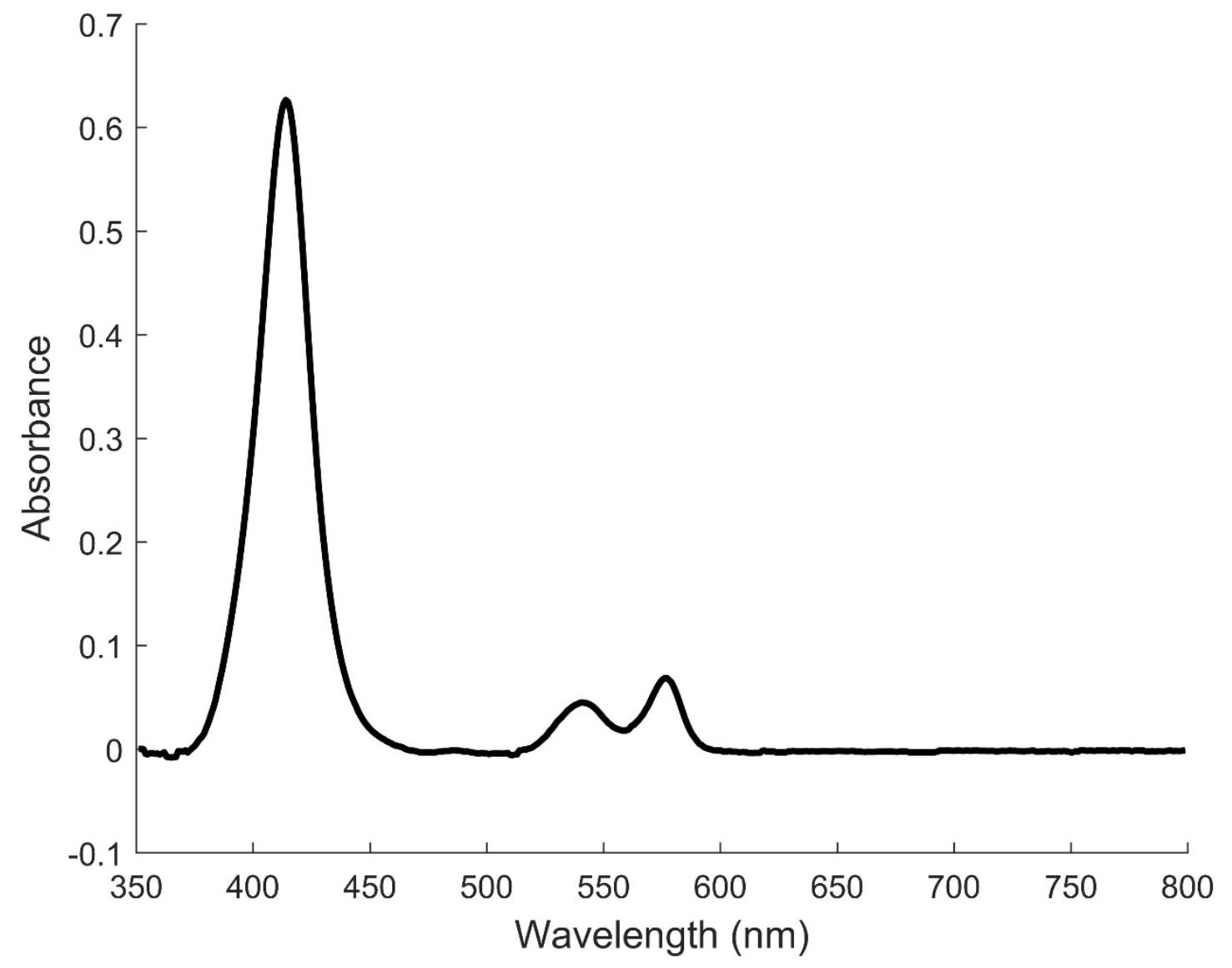

Figure 3: UV-VIS absorption spectrum of oxyhaemoglobin 


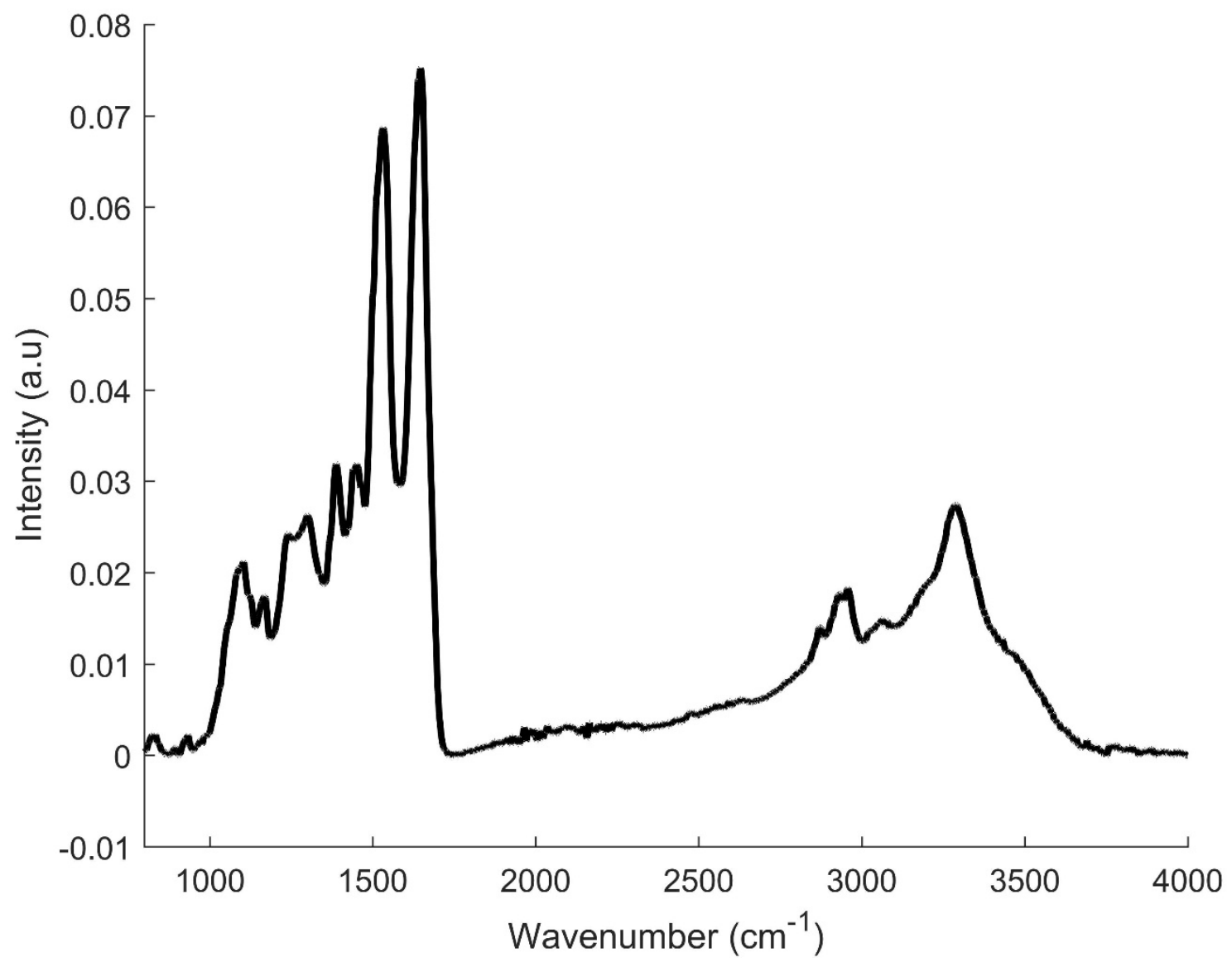

Figure 4: Mean and standard deviation of FTIR absorption spectrum of haemoglobin. 
A.

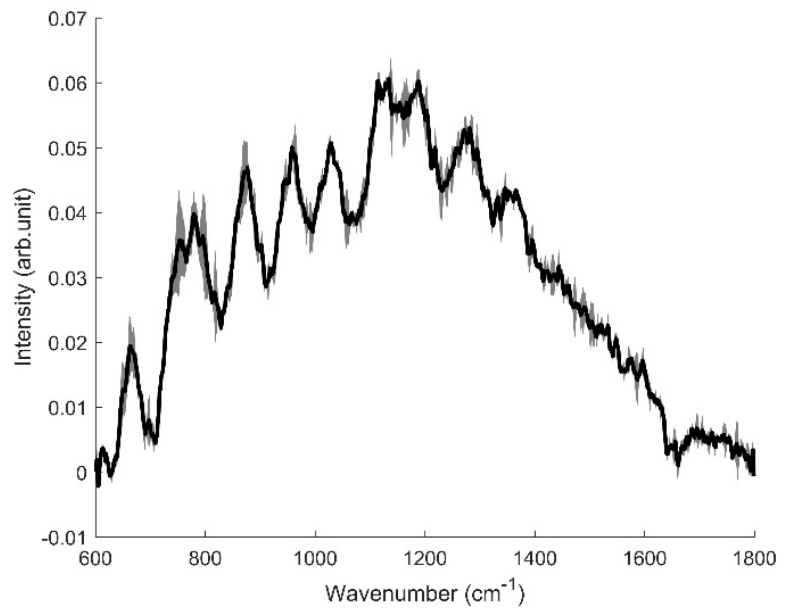

C.

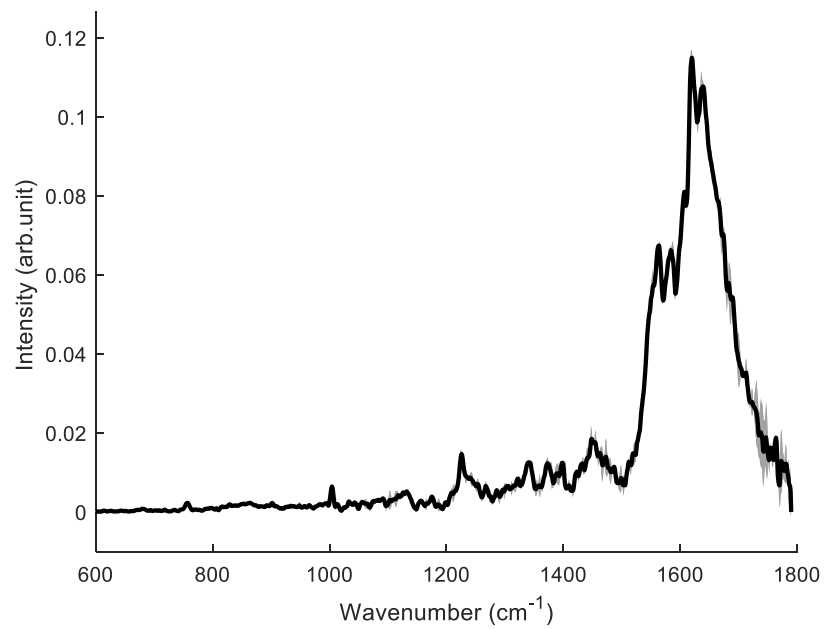

B.

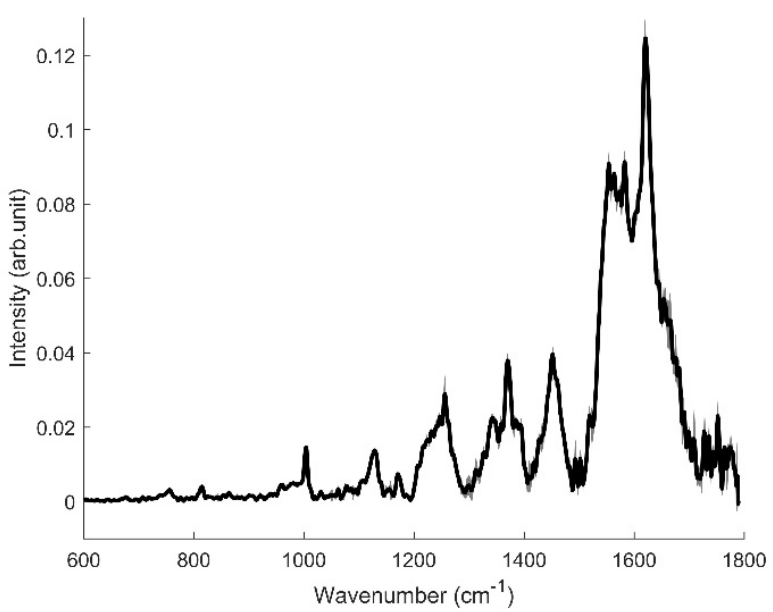

Figure 5: Mean and standard deviation Raman spectrum of (A) haemoglobin standard (B) diluted haemoglobin standard and (C) haemoglobin isolated from blood. 


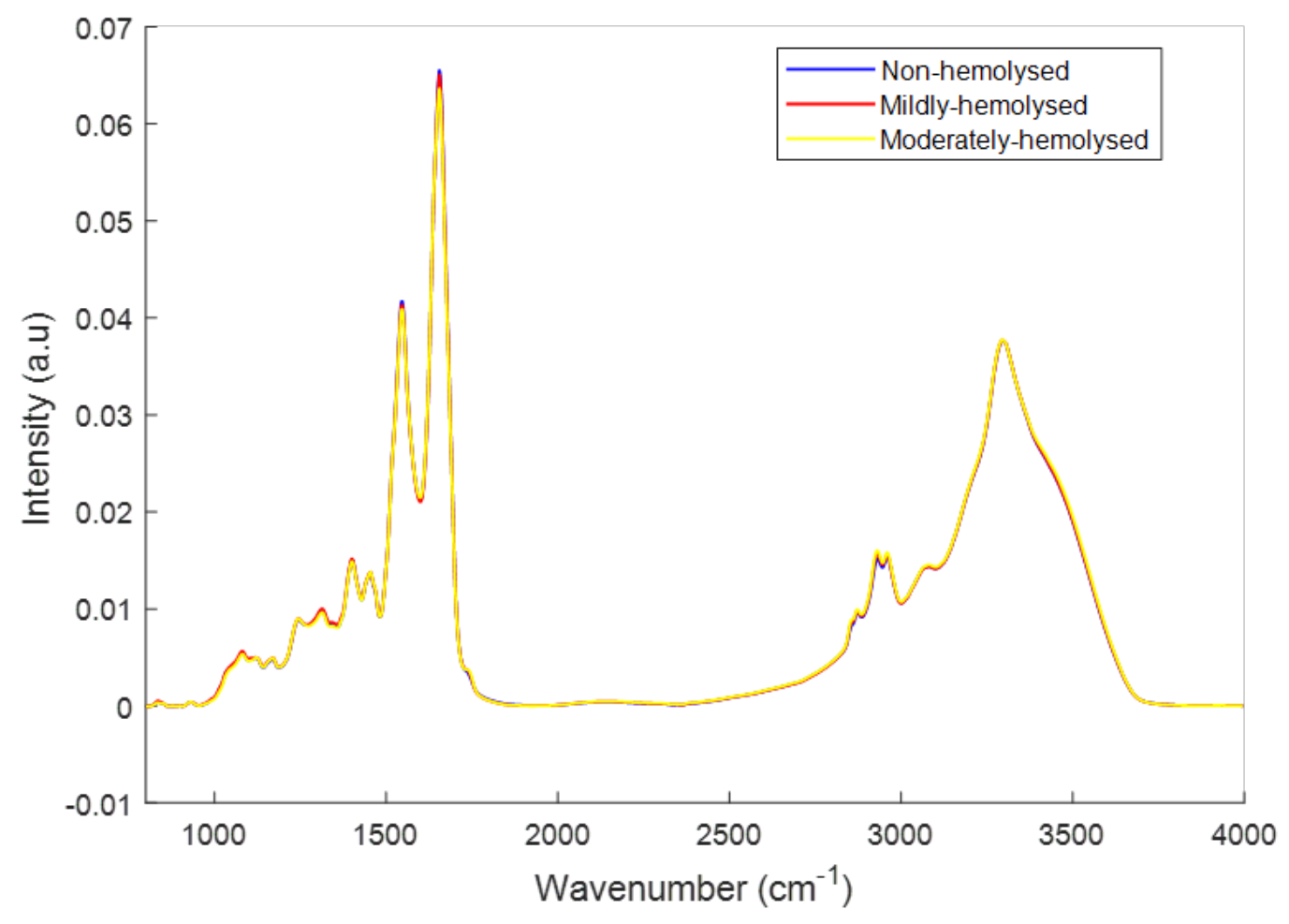

Figure 6: Mean FTIR spectra of non-haemolysed, mildly haemolysed and moderately haemolysed plasma samples. 

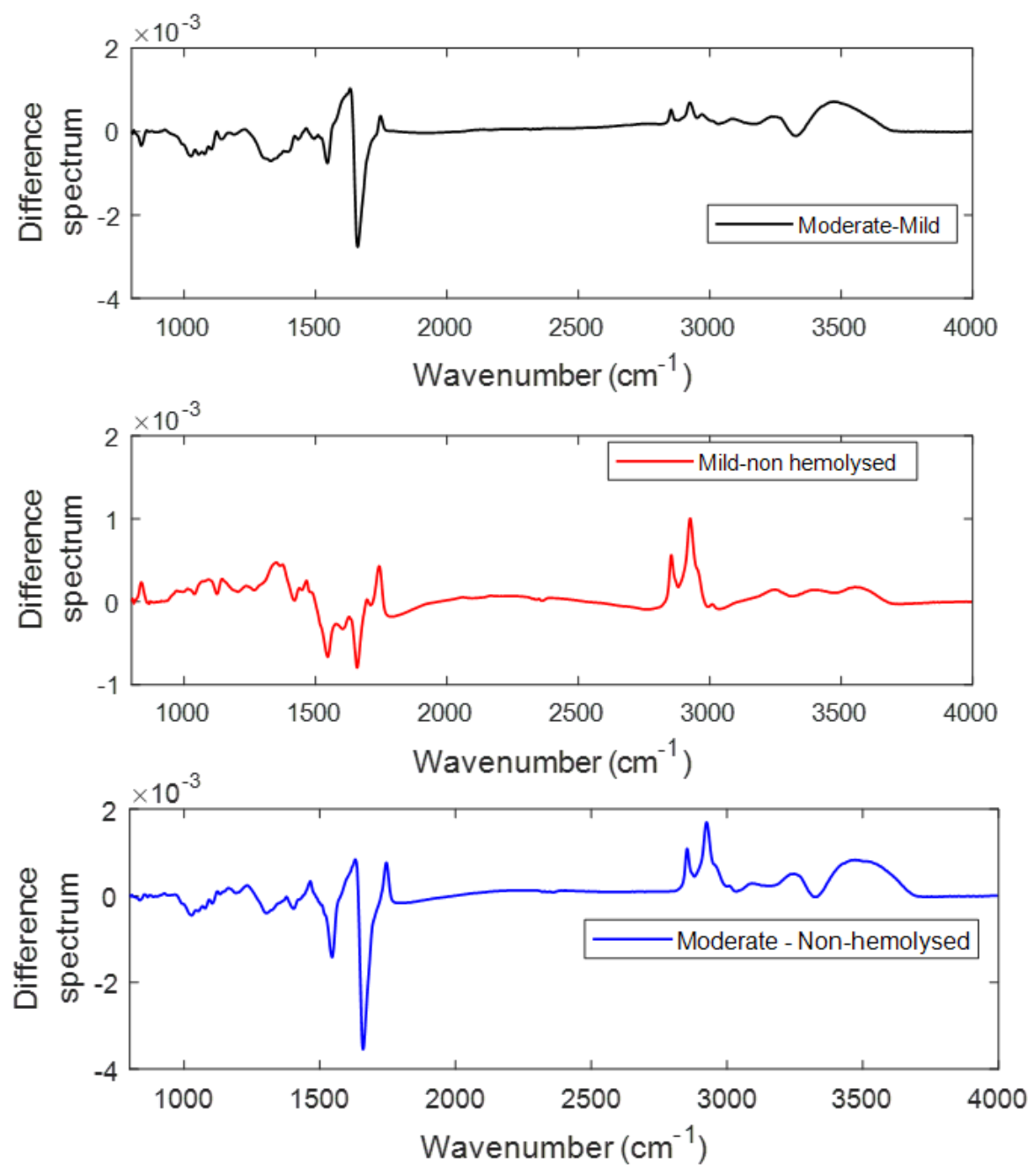

Figure 7: Difference FTIR spectra of non-haemolysed and haemolysed plasma groups. Difference spectra were computed by subtracting the mean FTIR spectra of haemolysed plasma from the mean FTIR spectra of non-haemolysed spectra. 


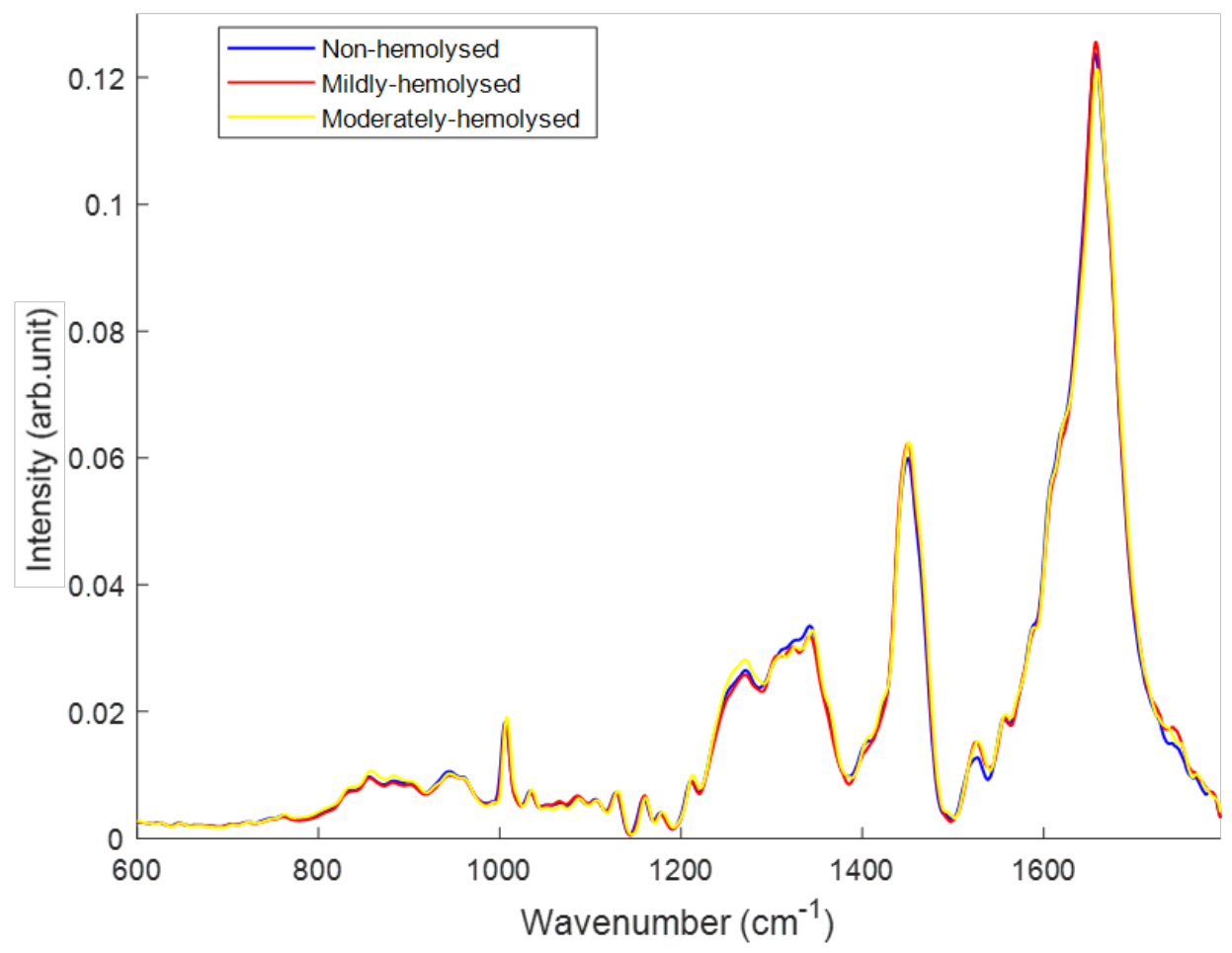

Figure 8: Mean Raman spectra of non-haemolysed, mildly-haemolysed and moderatelyhaemolysed plasma samples. 

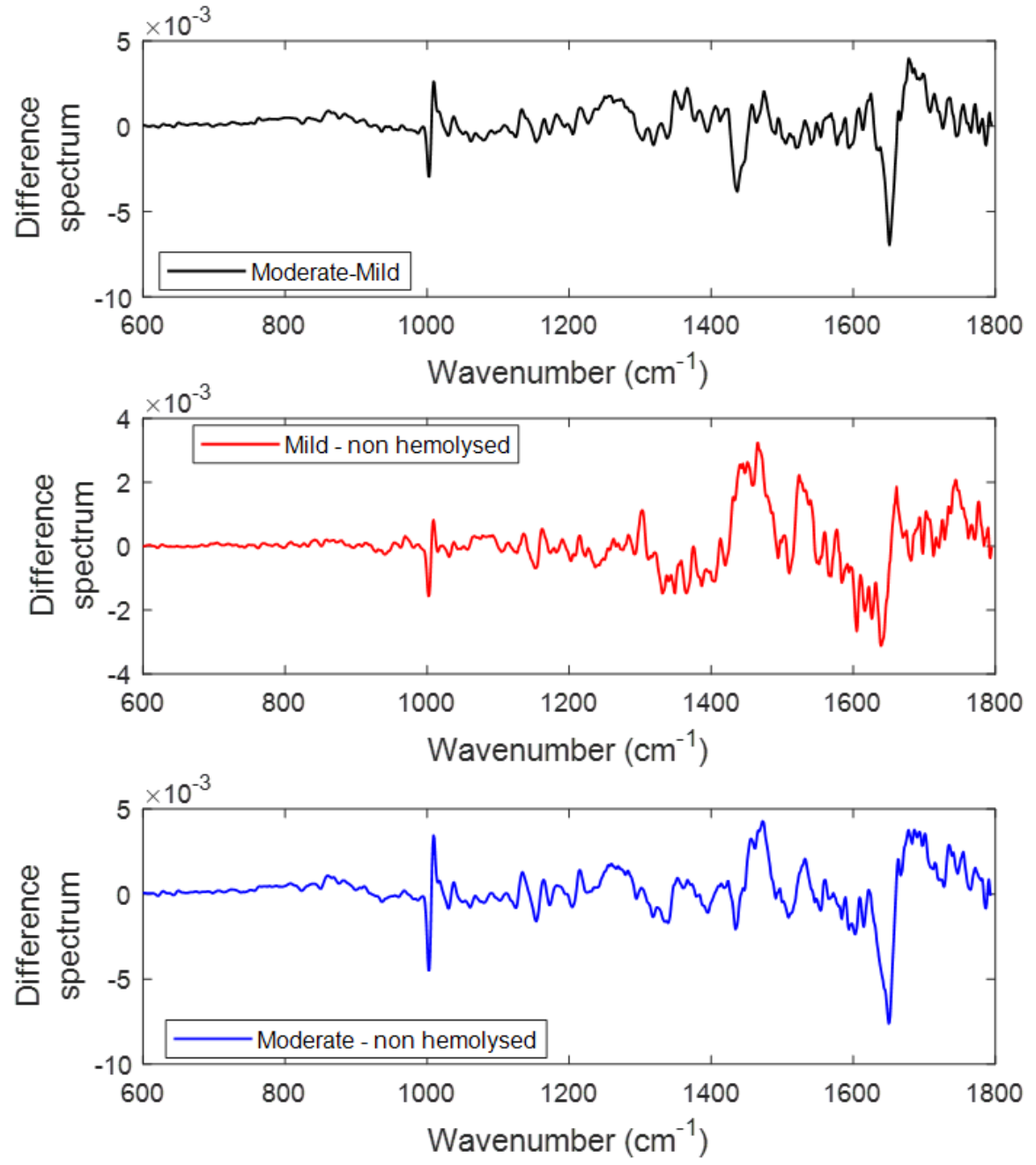

Figure 9: Difference Raman spectra of non-haemolysed and haemolysed plasma groups. Difference spectra were computed by subtracting the mean Raman spectra of haemolysed plasma from the mean Raman spectra of non-haemolysed spectra. 
A.

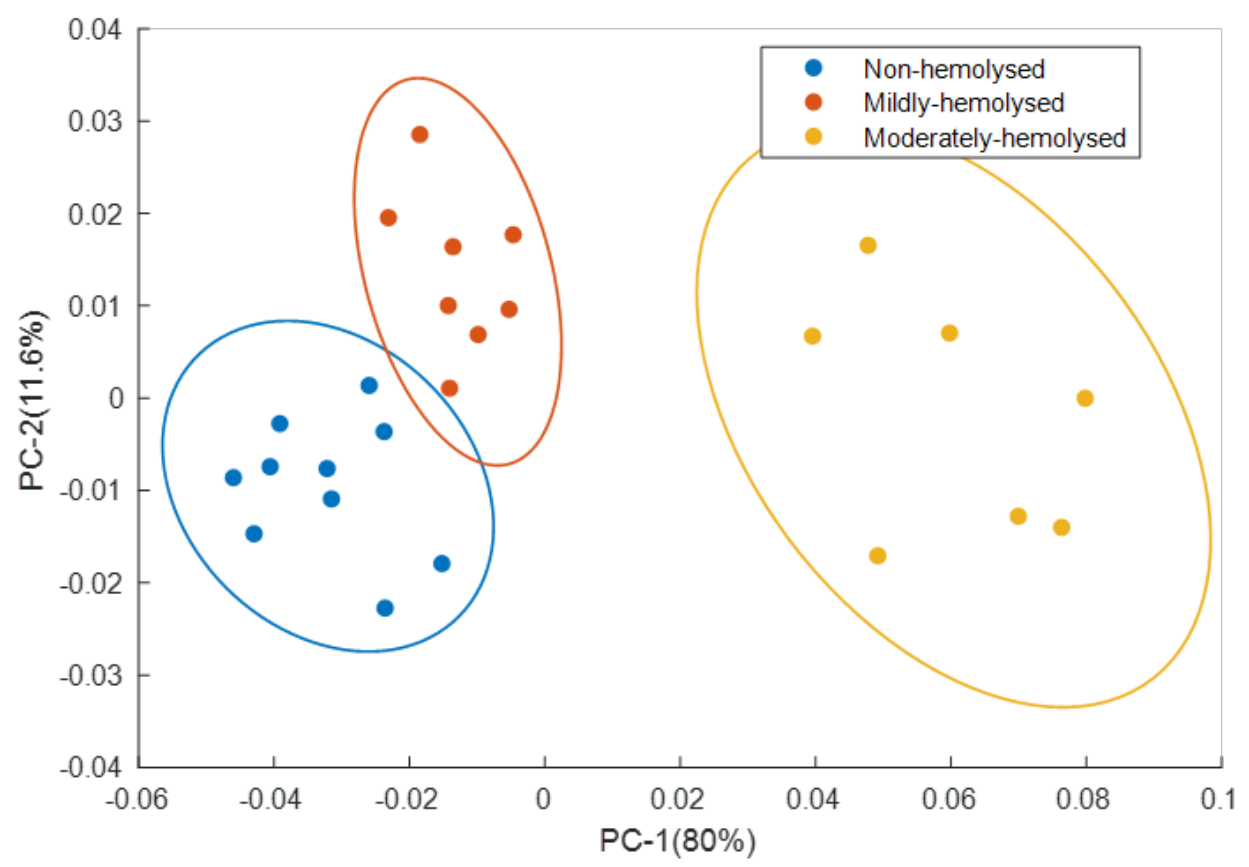

B.
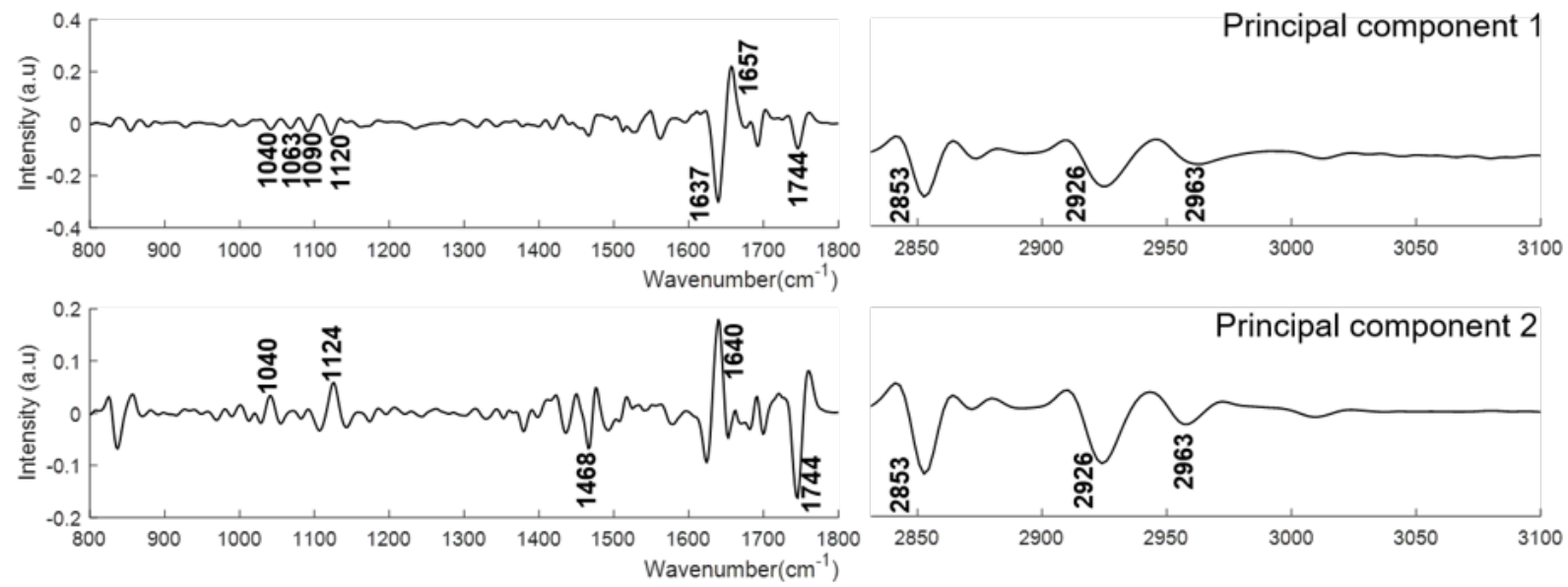

Figure 10: PCA for FTIR spectra of non-haemolysed, mildly-haemolysed and moderatelyhaemolysed plasma samples. (A) Score plots are shown for patients at non-haemolysed plasma (blue), mildly-hemolysed plasma (red), and moderately-hemolysed plasma (yellow (B) PC-1 and PC-2 loading plots for regions $800-1800 \mathrm{~cm}^{-1}$ and $2800-3100 \mathrm{~cm}^{-1}$. Covariance ellipse $(95 \%$ confidence) are shown for each class. 
A.

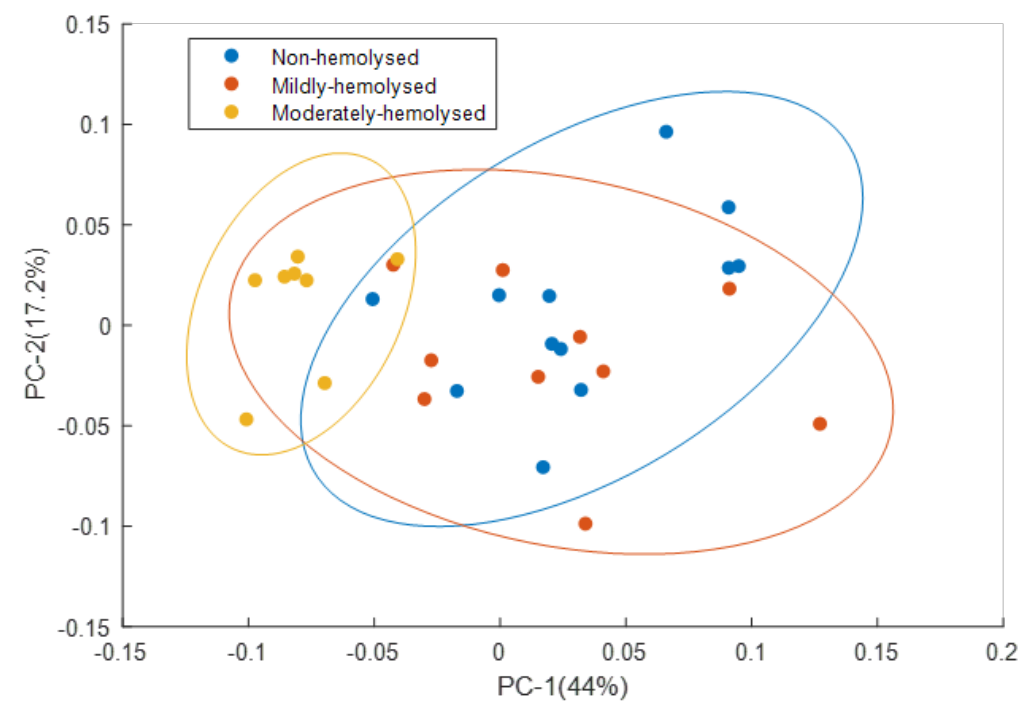

B.
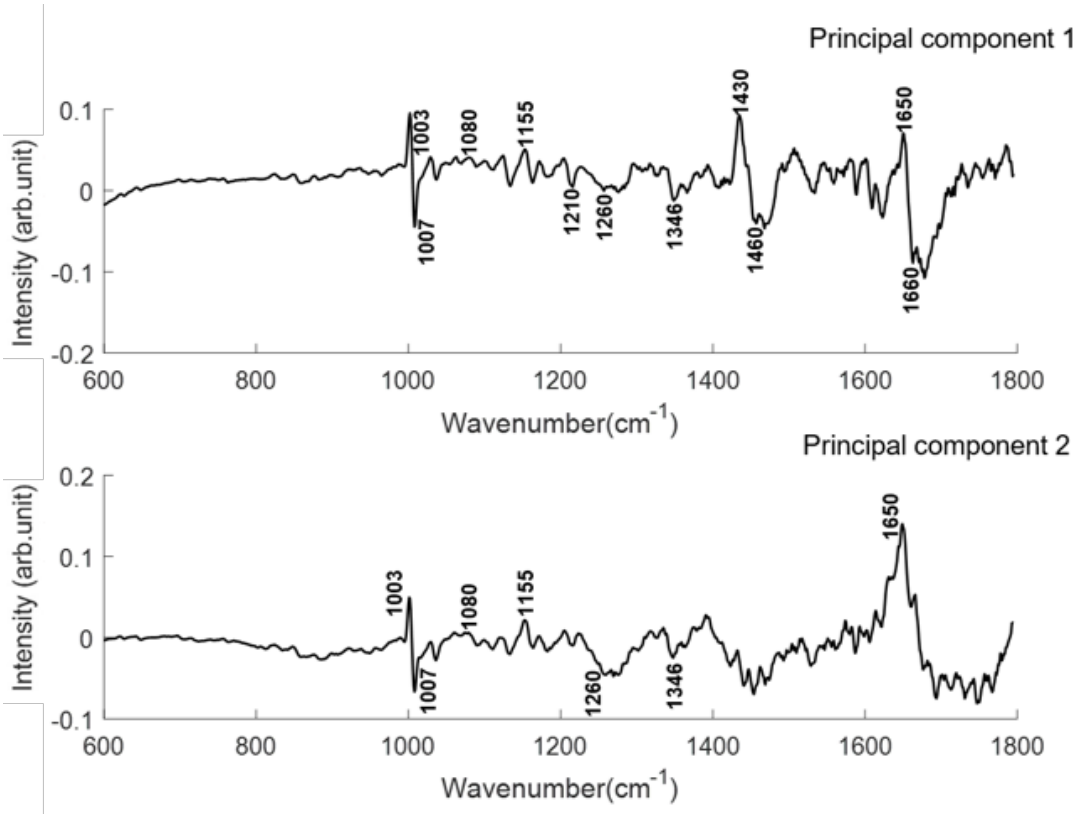

Figure 11: PCA for Raman spectra of non-haemolysed, mildly-haemolysed and moderatelyhaemolysed plasma samples. (A) Score plots are shown for patients at non-haemolysed plasma (blue), mildly-hemolysed plasma (red), and moderately-hemolysed plasma (yellow (B) PC-1 and PC-2 loading plots for regions $600-1800 \mathrm{~cm}^{-1}$. Covariance ellipse (95\% confidence) are shown for each class. 


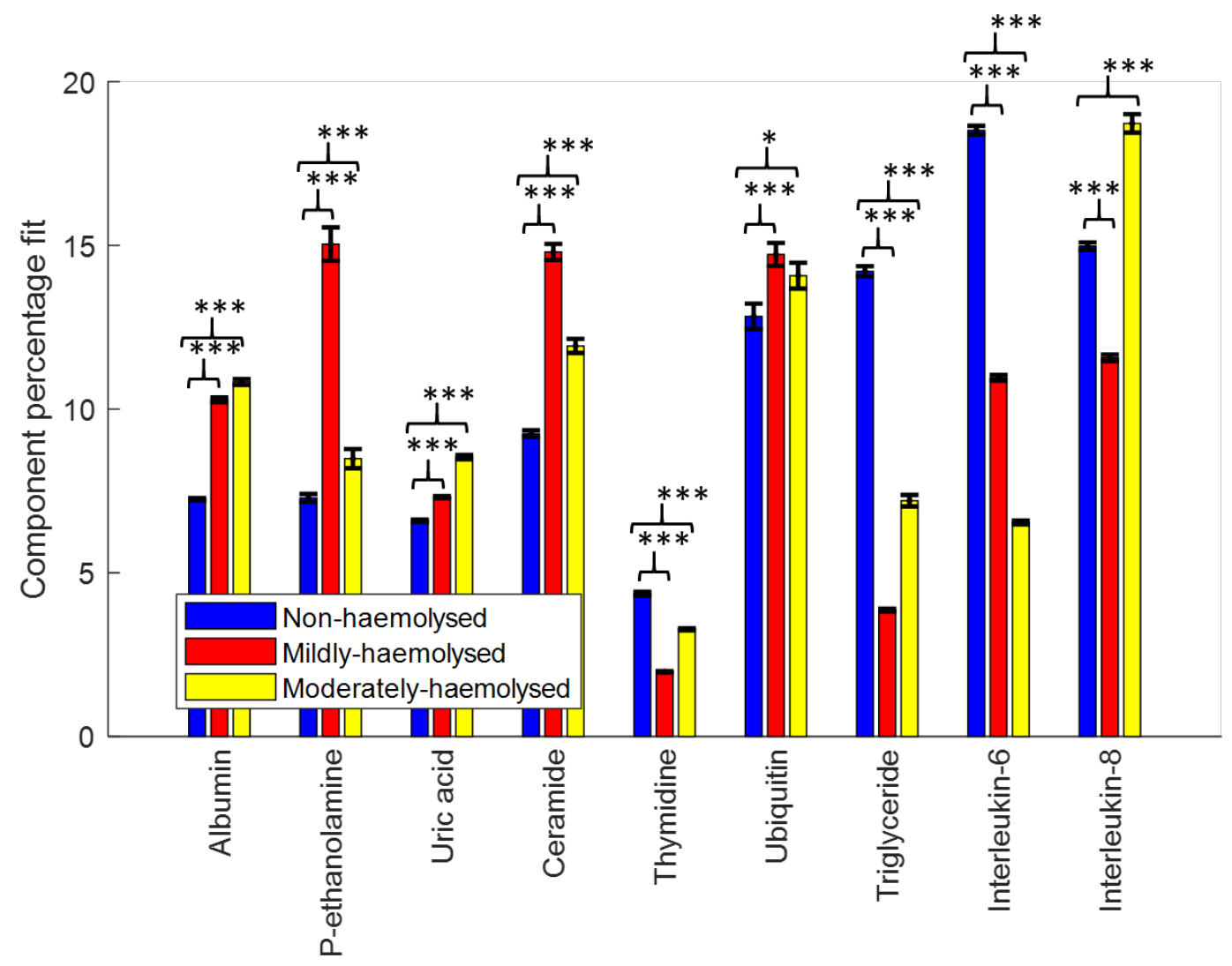

Figure 12: Relative concentrations of pure molecular reference species from least squares fit of FTIR spectra of non-haemolysed and haemolysed prostate cancer plasma samples. Error bars represent the standard error. Significance shown by $(*, * *$, and $* * *)$ indicates the significance of $p \leq 0.05, p \leq 0.01$ and $p \leq 0.001$ respectively. 


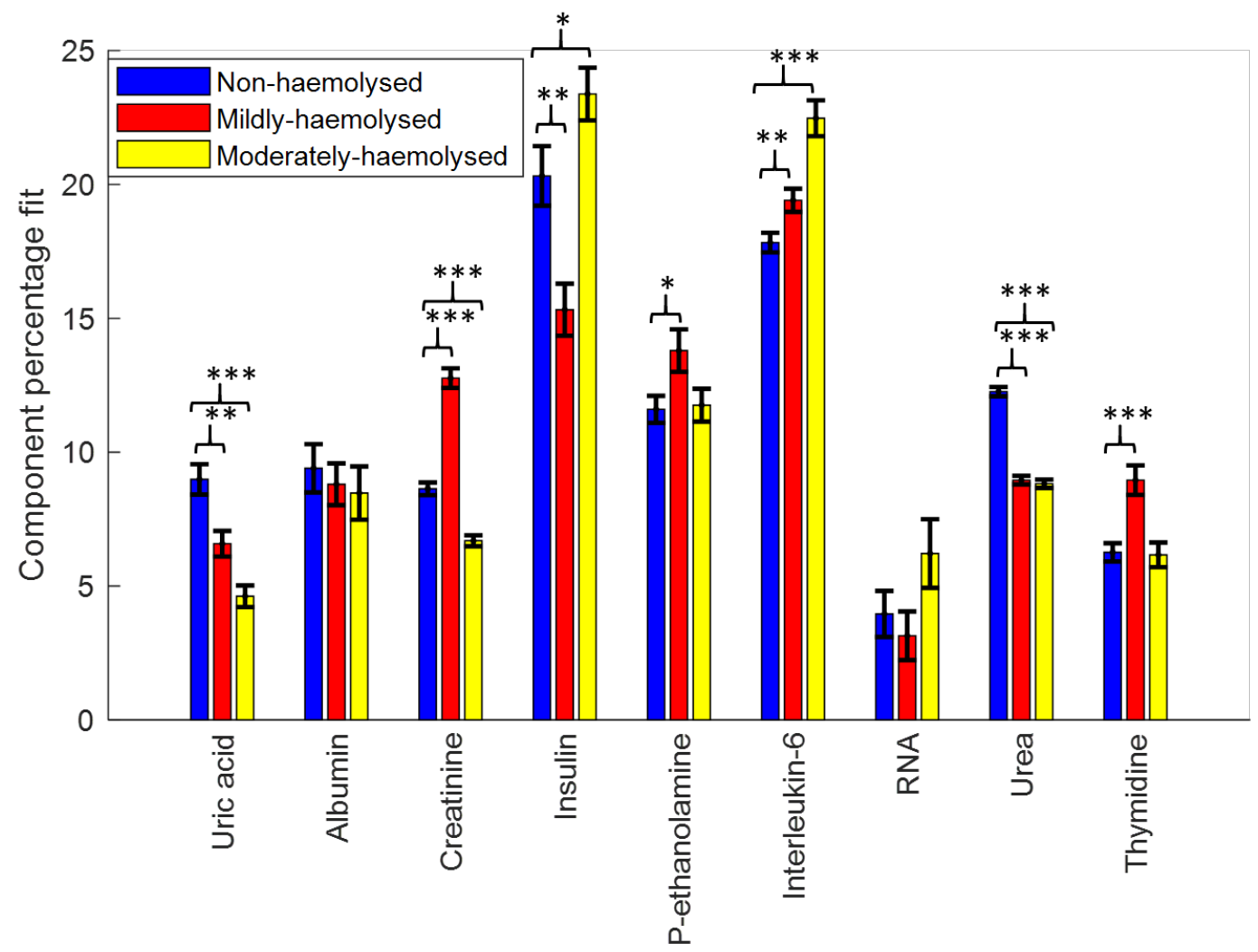

Figure 13: Relative concentrations of pure molecular reference species from least squares fit of Raman spectra of non-haemolysed and haemolysed prostate cancer plasma samples. Error bars represent the standard error. Significance shown by $(*, * *$, and $* * *)$ indicates the significance of $p \leq 0.05, p \leq 0.01$ and $p \leq 0.001$ respectively. 


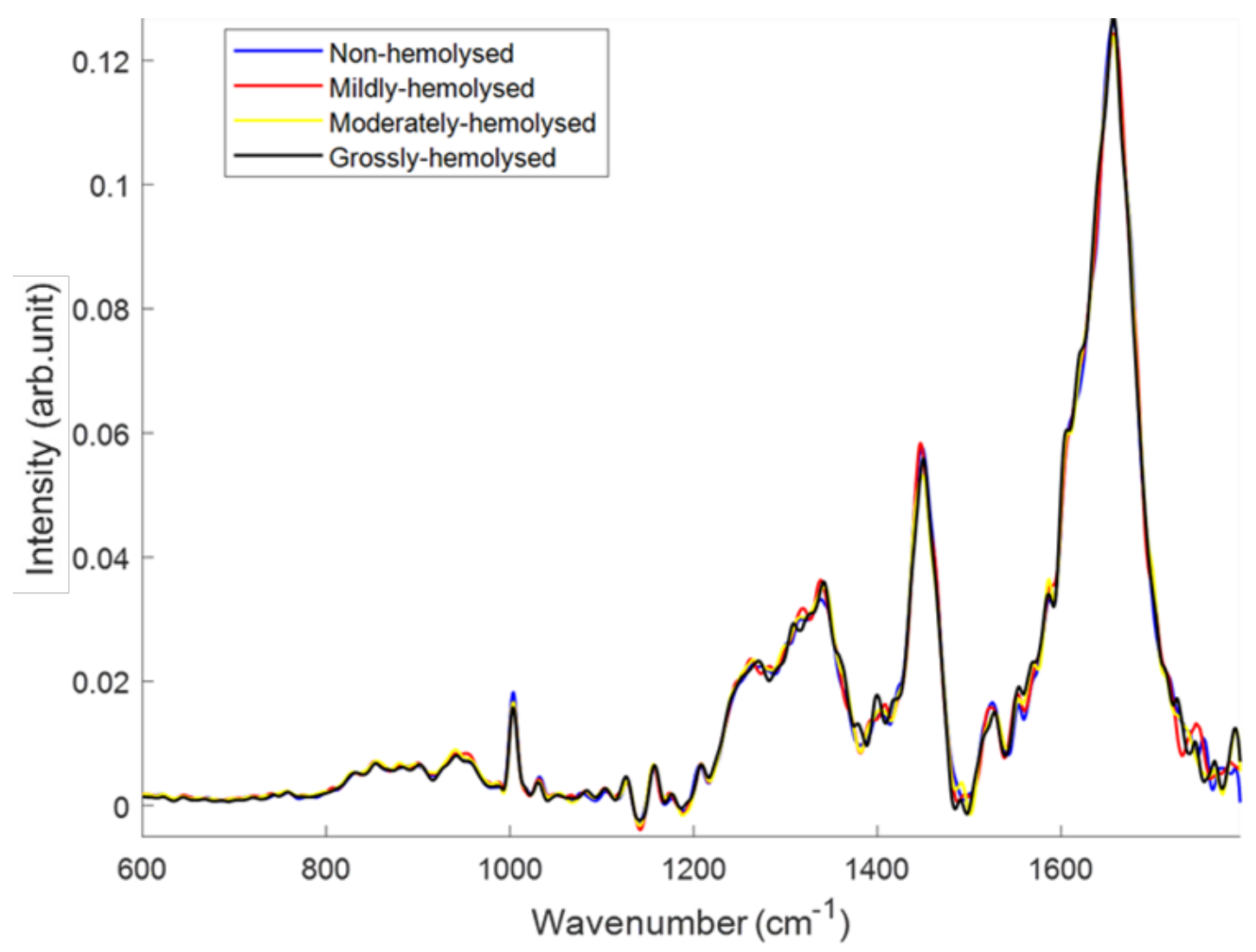

Figure 14: Mean Raman spectra of non-haemolysed and in vitro haemolysed plasma samples. 


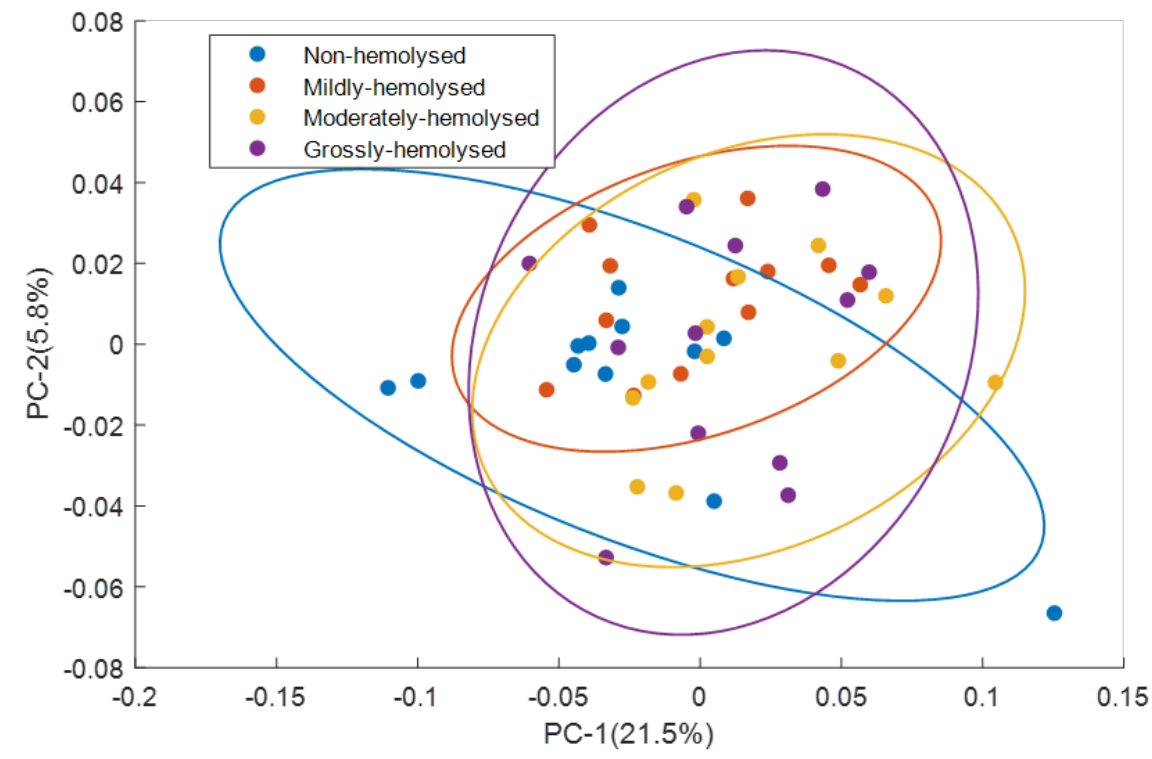

Figure 15: PCA scatter plot for Raman spectra of non-haemolysed and in vitro haemolysed plasma samples. Covariance ellipse (95\% confidence) are shown for each class. 


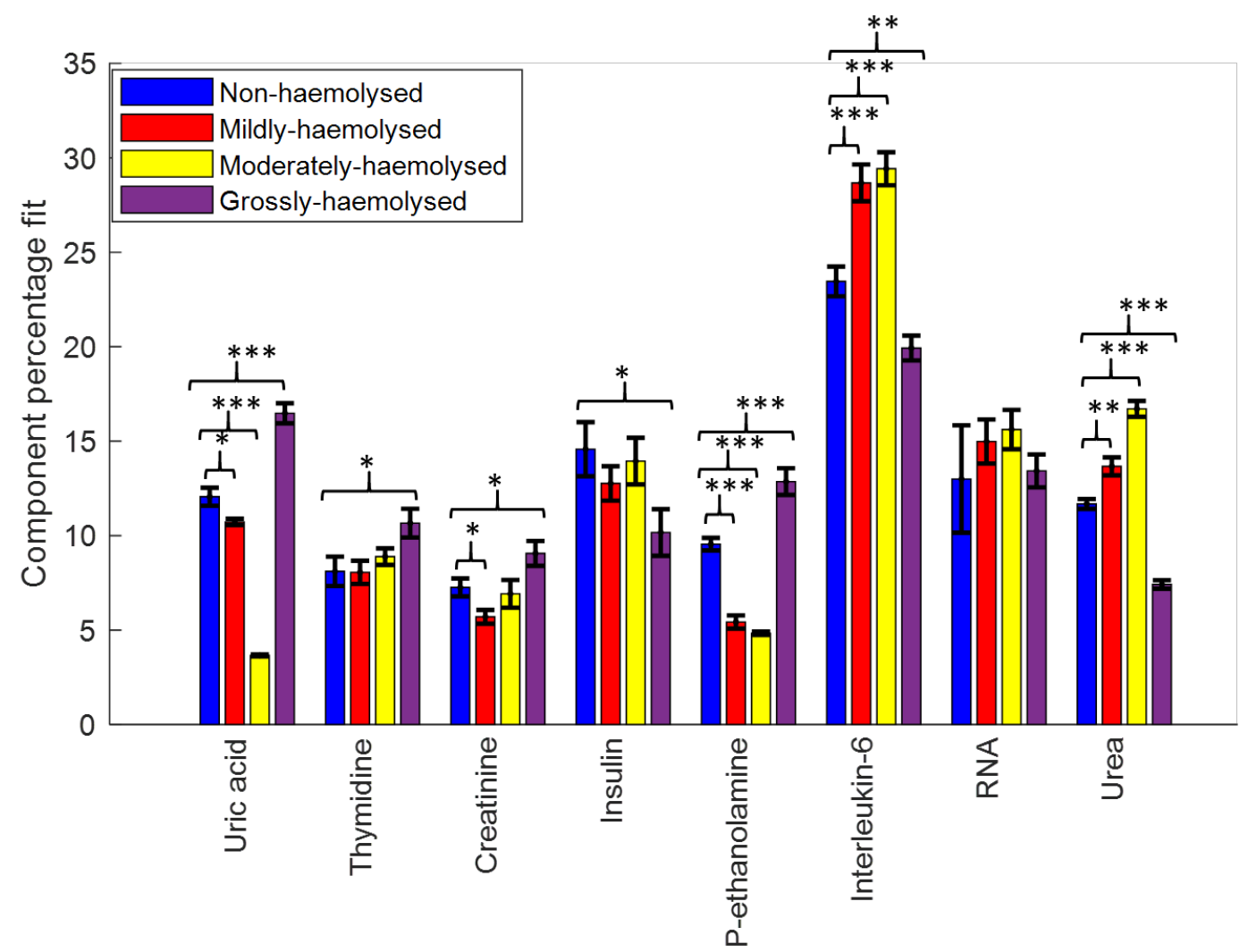

Figure 16: Relative concentrations of pure molecular reference species from least squares fit of Raman spectra of non-haemolysed and in vitro haemolysed plasma samples. Error bars represent the standard error. Significance shown by $(*, * *$, and $* * *)$ indicates the significance of $p \leq 0.05$, $p \leq 0.01$ and $p \leq 0.001$ respectively. 


\section{Supplementary material}

Table S1: Details of pure molecular reference species used in this study.

\begin{tabular}{|c|c|c|c|c|}
\hline SN & Component name & Company & Product number & State \\
\hline \multicolumn{5}{|c|}{ Protein and related species } \\
\hline 1 & Albumin & Sigma Aldrich & A7030 & Powder \\
\hline 2 & Haemoglobin & Sigma Aldrich & H7379 & Powder \\
\hline 3 & Interleukin-6 & Sigma Aldrich & I13965 & Powder \\
\hline 4 & Interleukin-8 & Sigma Aldrich & $\mathrm{I}-1645$ & Powder \\
\hline 5 & Ubiquitin & Sigma Aldrich & U6253 & Powder \\
\hline 6 & Apolipoprotein E4 & Sigma Aldrich & A3234 & Powder \\
\hline \multicolumn{5}{|c|}{ Nucleic acids and nucleobases } \\
\hline 7 & RNA & Sigma Aldrich & R6750 & Powder \\
\hline 8 & DNA & Sigma Aldrich & D-1626 & Powder \\
\hline 9 & Thymidine & Sigma Aldrich & T1895 & Powder \\
\hline \multicolumn{5}{|c|}{ Lipids, fatty acids and related species } \\
\hline 10 & Ceramide & Sigma Aldrich & 22244 & Powder \\
\hline 11 & Cholesterol & Sigma Aldrich & C3045 & Powder \\
\hline 12 & Linoleic acid & Sigma Aldrich & L1376 & Liquid \\
\hline 13 & Linolenic acid & Sigma Aldrich & L2376 & Liquid \\
\hline 14 & Oleic acid & Sigma Aldrich & 01008 & Liquid \\
\hline 15 & L- $\alpha$-Phosphatidylcholine & Sigma Aldrich & L4129 & Powder \\
\hline 16 & L- $\alpha$-Phosphatidylserine & Avanti & $8703363 \mathrm{C}$ & Liquid \\
\hline 17 & L- $\alpha$-Phosphatidylinositol & Sigma Aldrich & P-6636 & Powder \\
\hline 18 & L- $\alpha$-Phosphatidylethanolamine & Sigma Aldrich & P-7693 & Powder \\
\hline 19 & Prostaglandin E1 & Sigma Aldrich & P5515 & Liquid \\
\hline 20 & PUFA & SUPELCO & $47015-U$ & Liquid \\
\hline 21 & Sphingomyelin & Sigma Aldrich & S0756 & Powder \\
\hline 22 & Triglyceride mixture & Sigma Aldrich & 17811-1AMP & Powder \\
\hline 23 & Arachidonic acid & Sigma Aldrich & 10931 & Liquid \\
\hline
\end{tabular}




\begin{tabular}{|c|c|c|c|c|}
\hline \multicolumn{5}{|c|}{ Other metabolites } \\
\hline 24 & $\beta$-Carotene & Sigma Aldrich & C4582 & Powder \\
\hline 25 & Urea & Sigma Aldrich & U4884 & Powder \\
\hline 26 & Uric acid & Sigma Aldrich & U2625 & Powder \\
\hline 27 & Creatinine & Sigma Aldrich & C4255 & Powder \\
\hline 28 & Glucose & Sigma Aldrich & G8270 & Powder \\
\hline 29 & Glycogen & Sigma Aldrich & G0885 & Powder \\
\hline 30 & Insulin & Sigma Aldrich & I2643 & Powder \\
\hline
\end{tabular}


Figure S1: Raman spectrum of pure molecular reference species.
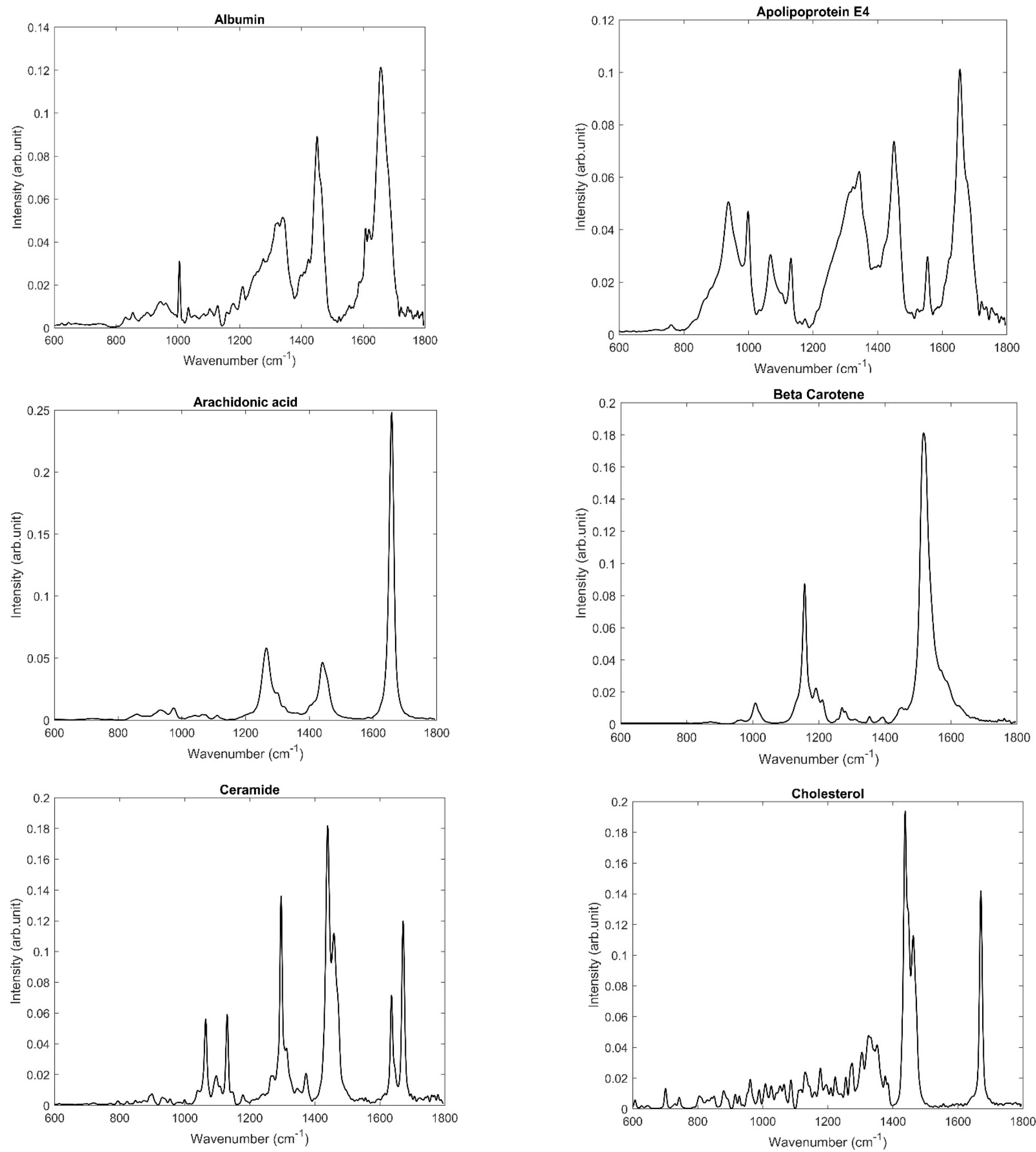

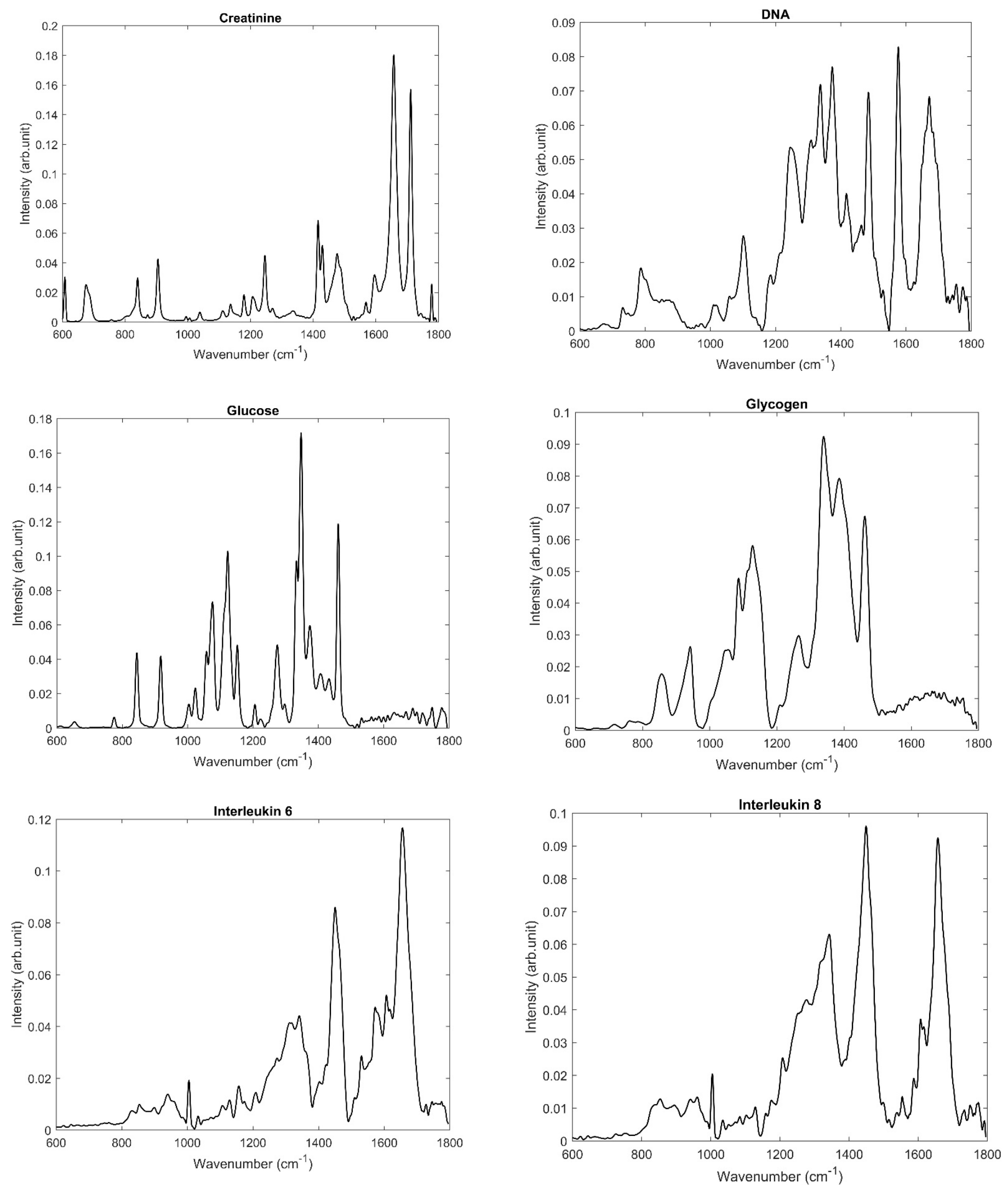

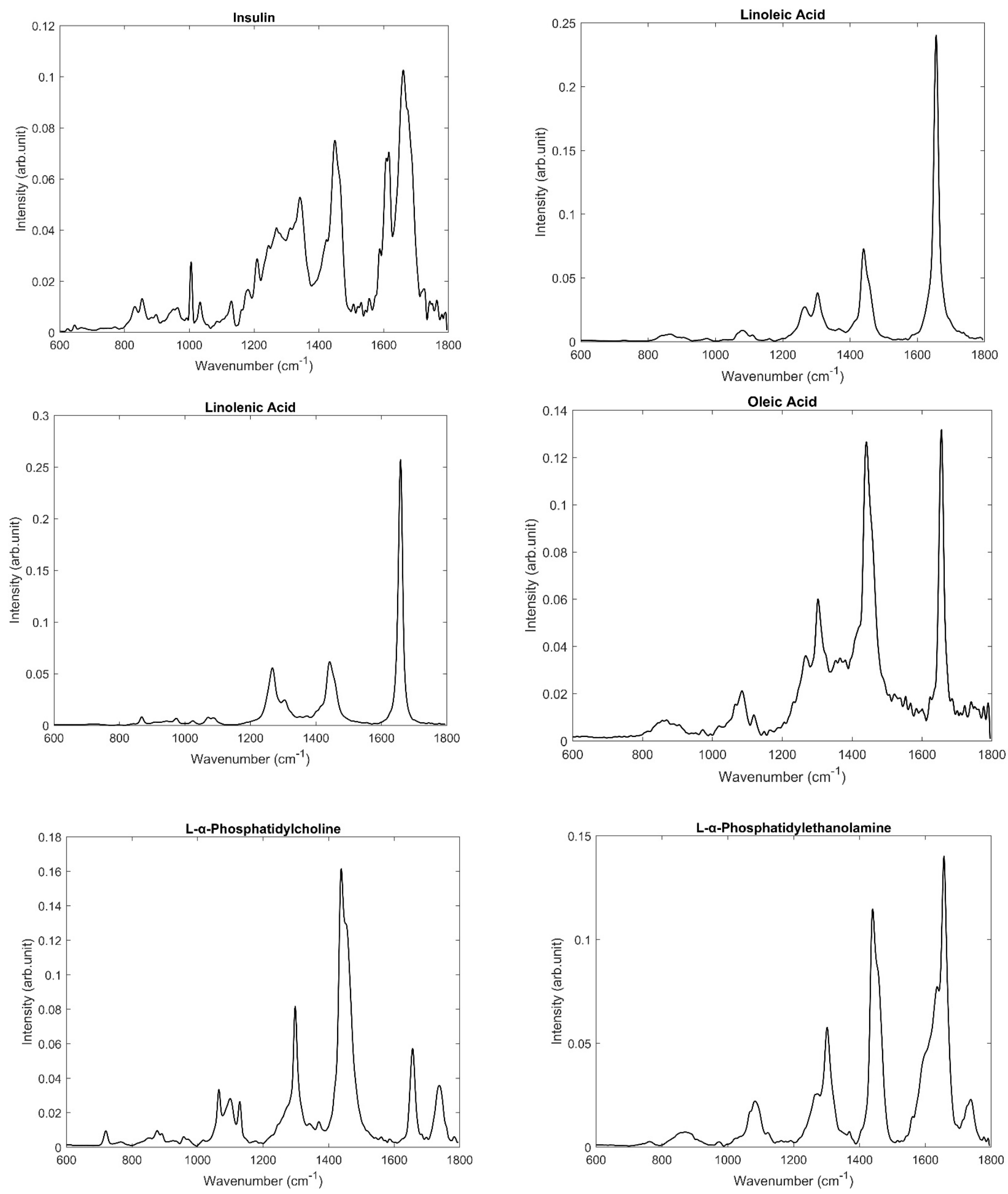



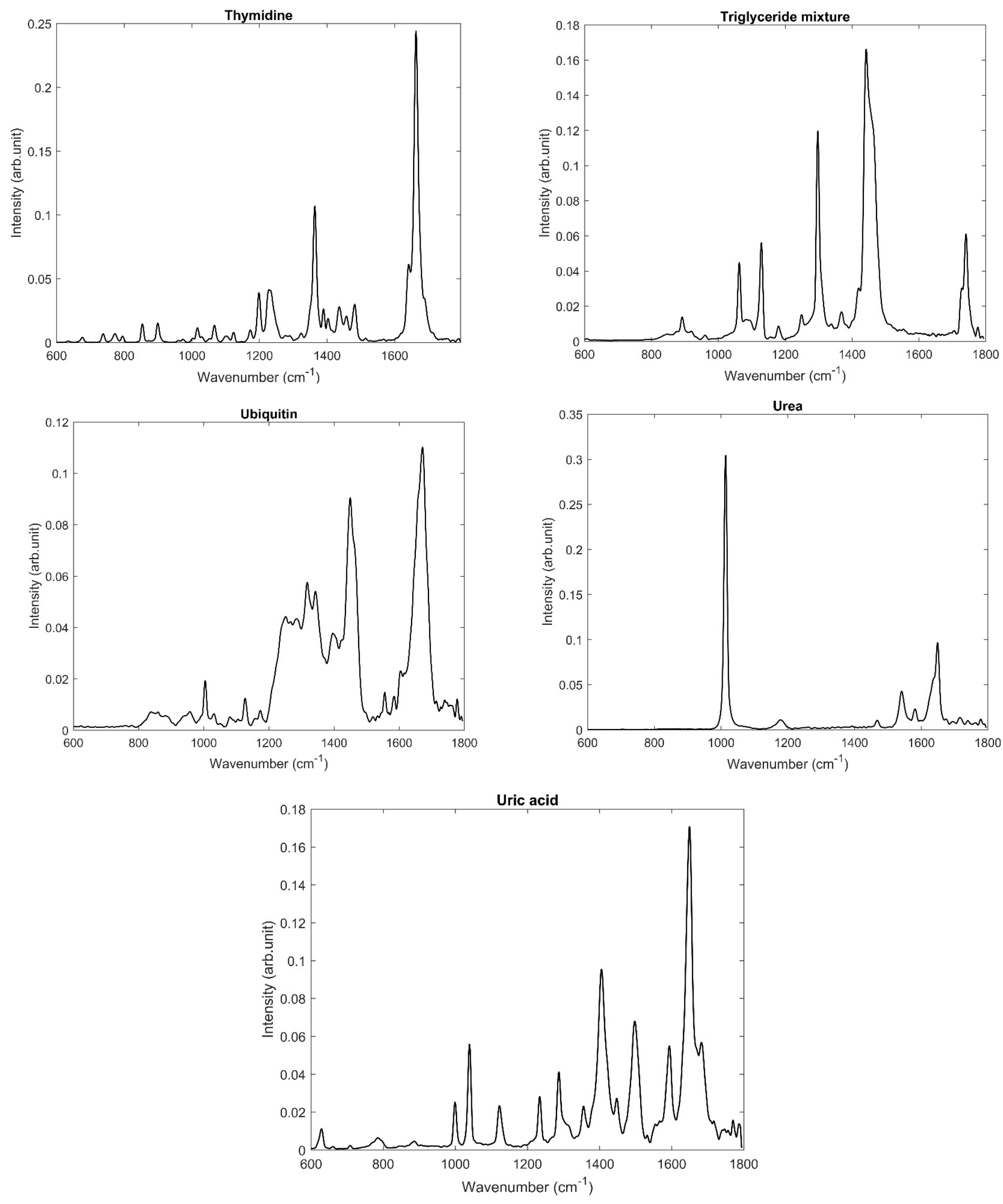
Figure S2: FTIR spectrum of pure molecular reference species.
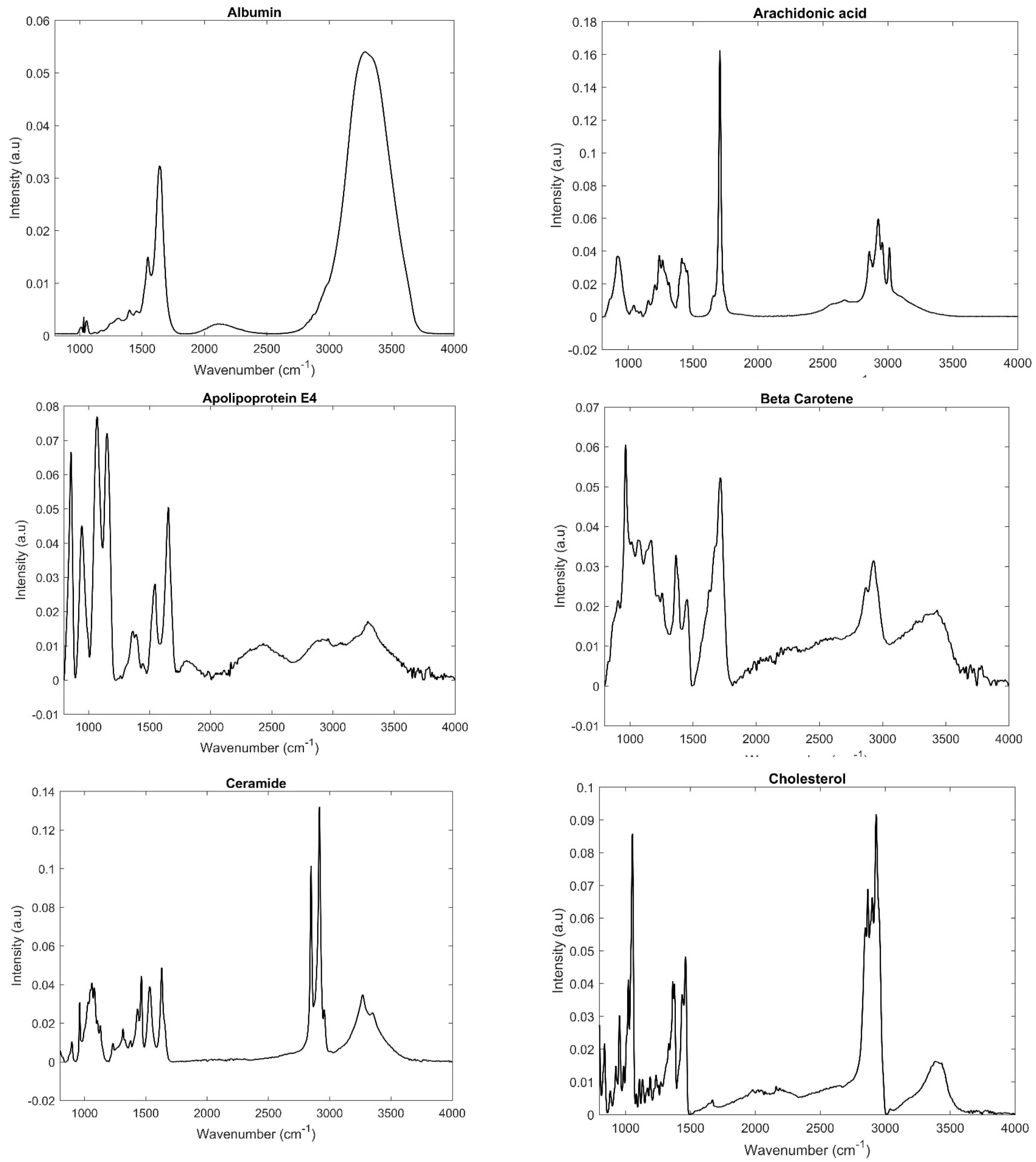

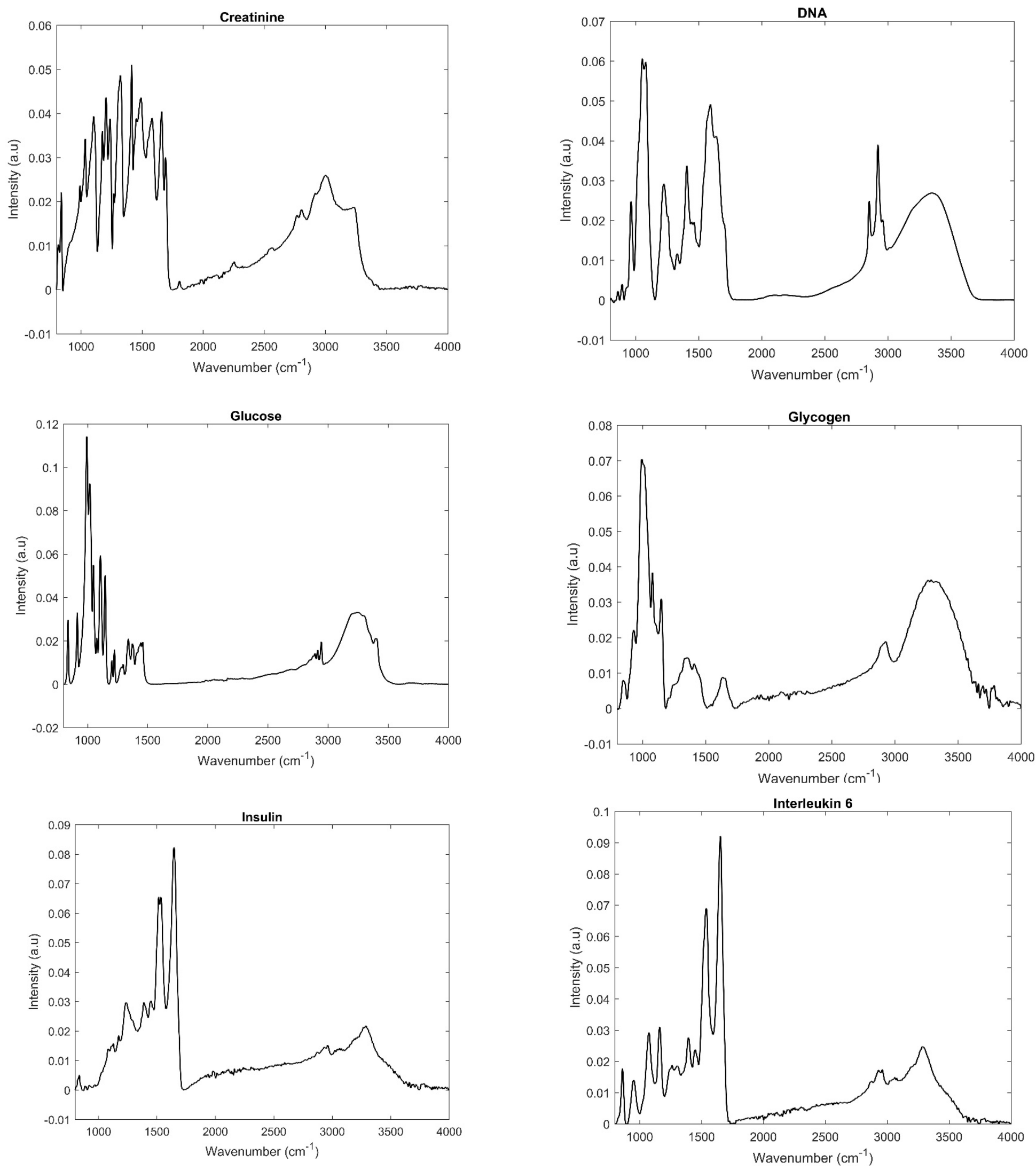

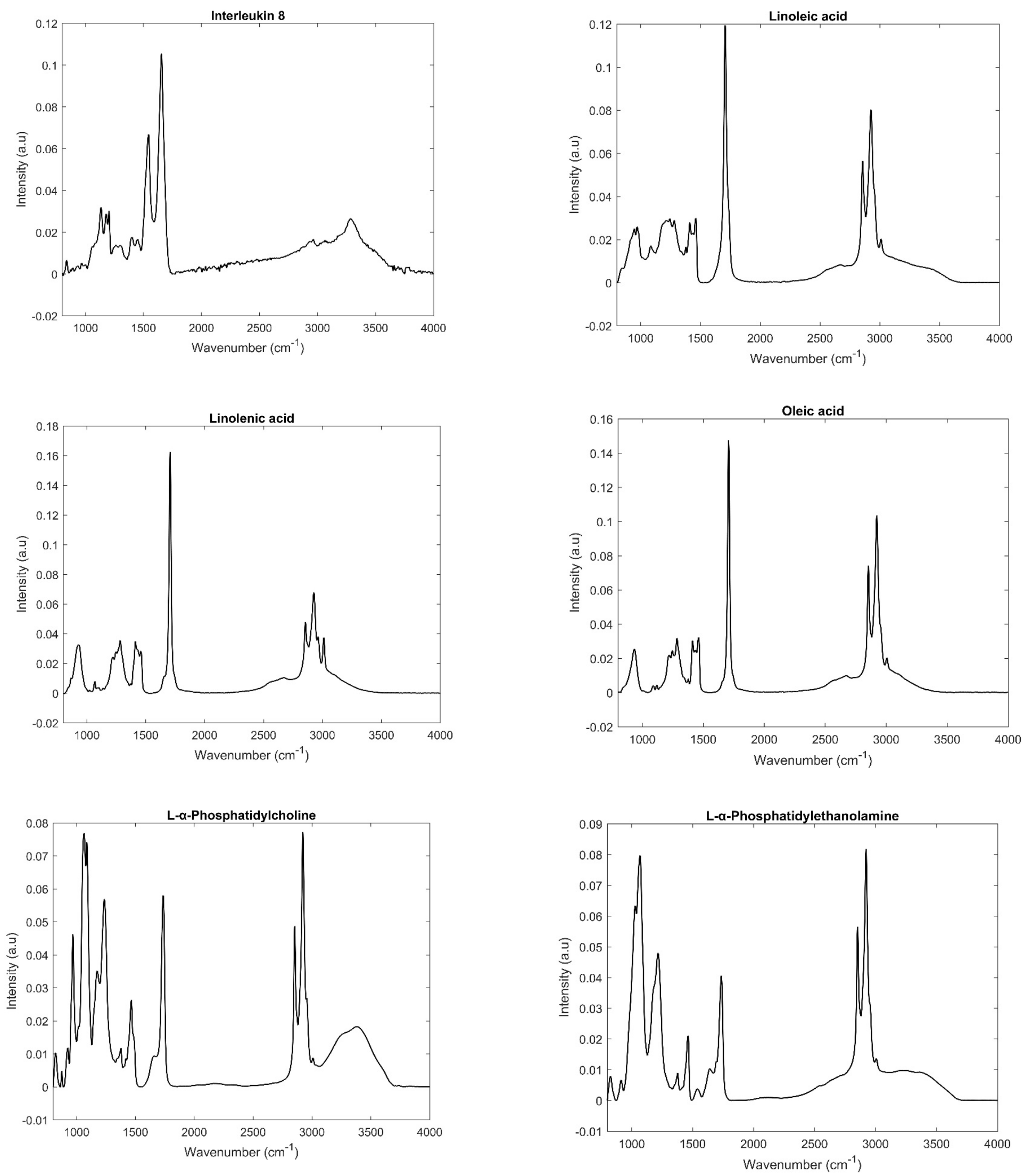

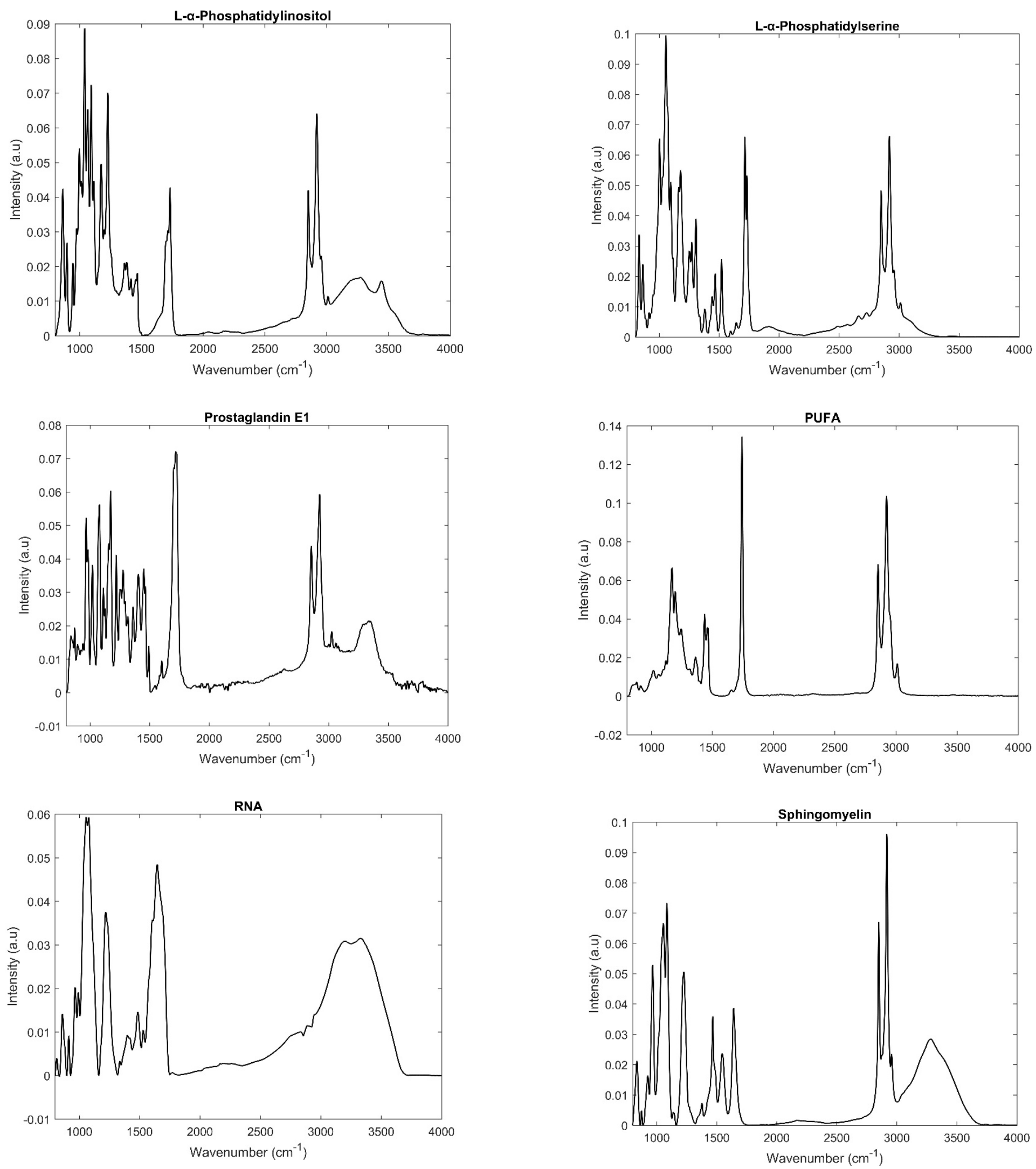

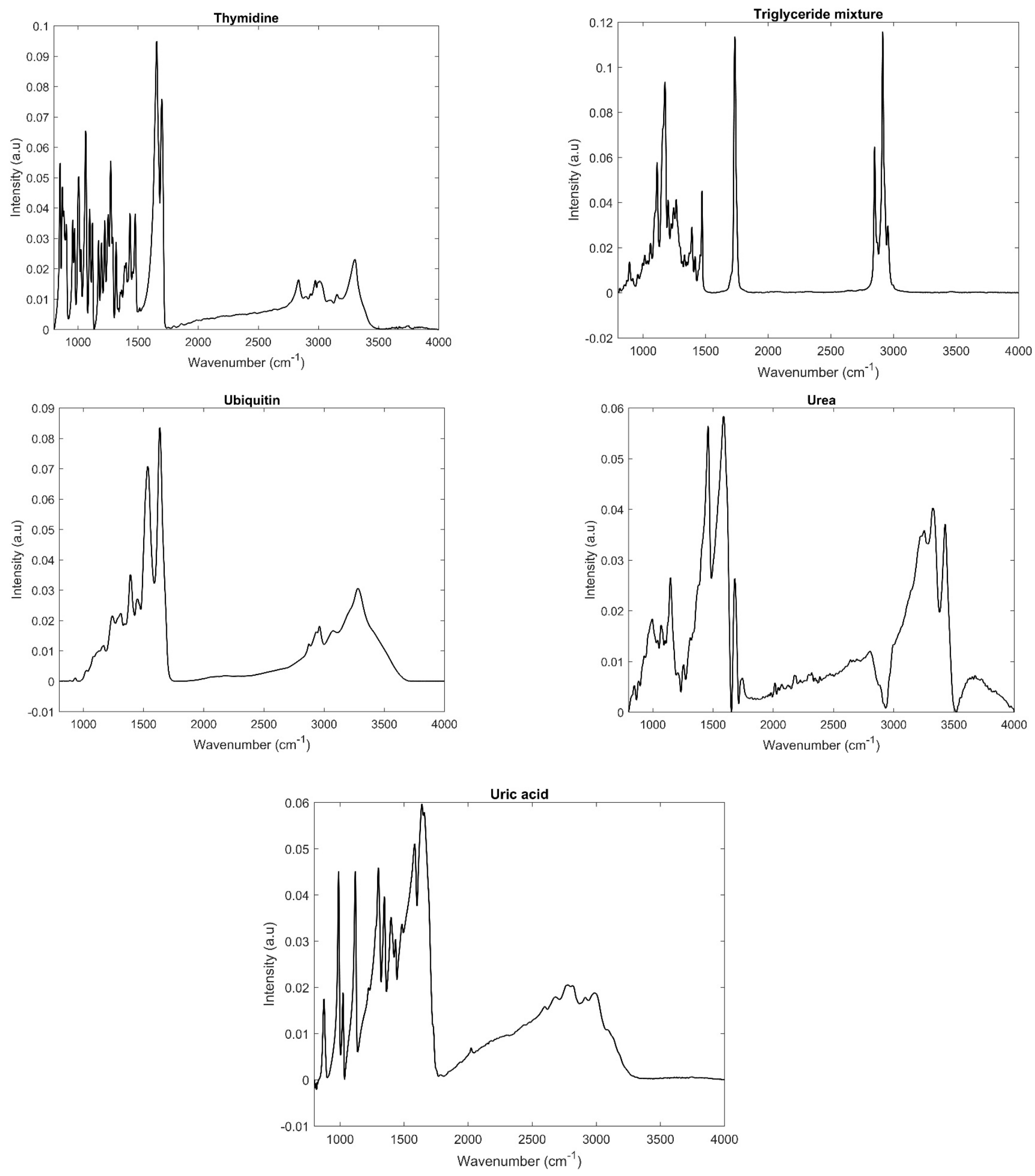